\title{
Emerging point-of-care biosensors for rapid diagnosis of COVID-19: current progress, challenges, and future prospects
}

\author{
Yousef Rasmi ${ }^{1,2}$ - Xiaokang $\mathrm{Li}^{3,4}$ - Johra Khan ${ }^{5}$ - Tugba Ozer ${ }^{6}$ - Jane Ru Choi ${ }^{7,8}$ \\ Received: 13 March 2021 / Accepted: 26 April 2021 / Published online: 18 May 2021 \\ (C) Springer-Verlag GmbH Germany, part of Springer Nature 2021
}

\begin{abstract}
Coronavirus disease 2019 (COVID-19) pandemic is currently a serious global health threat. While conventional laboratory tests such as quantitative real-time polymerase chain reaction (qPCR), serology tests, and chest computerized tomography (CT) scan allow diagnosis of COVID-19, these tests are time-consuming and laborious, and are limited in resource-limited settings or developing countries. Point-of-care (POC) biosensors such as chip-based and paper-based biosensors are typically rapid, portable, cost-effective, and user-friendly, which can be used for COVID-19 in remote settings. The escalating demand for rapid diagnosis of COVID-19 presents a strong need for a timely and comprehensive review on the POC biosensors for COVID-19 that meet ASSURED criteria: Affordable, Sensitive, Specific, User-friendly, Rapid and Robust, Equipment-free, and Deliverable to end users. In the present review, we discuss the importance of rapid and early diagnosis of COVID-19 and pathogenesis of COVID-19 along with the key diagnostic biomarkers. We critically review the most recent advances in POC biosensors which show great promise for the detection of COVID-19 based on three main categories: chip-based biosensors, paper-based biosensors, and other biosensors. We subsequently discuss the key benefits of these biosensors and their use for the detection of antigen, antibody, and viral nucleic acids. The commercial POC biosensors for COVID-19 are critically compared. Finally, we discuss the key challenges and future perspectives of developing emerging POC biosensors for COVID-19. This review would be very useful for guiding strategies for developing and commercializing rapid POC tests to manage the spread of infections.
\end{abstract}

Keywords COVID-19 · Point-of-care biosensors · Chip-based biosensors · Paper-based biosensors · Diagnostic biomarkers . Commercialization

\section{Introduction}

Coronavirus disease 2019 (COVID-19) has currently become a global health threat [1,2]. The number of COVID-19 cases continues to rise drastically across the globe, affecting more than 200 countries and territories, with over 50 million

Jane Ru Choi

janeruchoi@gmail.com

1 Department of Biochemistry, Faculty of Medicine, Urmia University of Medical Sciences, 5714783734, Urmia, Iran

2 Cellular and Molecular Research Center, Urmia University of Medical Sciences, 5714783734, Urmia, Iran

3 Ludwig Institute for Cancer Research, University of Lausanne, Agora Center, 1005 Lausanne, Switzerland

4 Department of Oncology, Centre hospitalier universitaire vaudois (CHUV), 1011 Lausanne, Switzerland confirmed cases and 1,000,000 deaths as of November 1, 2020 [1]. Generally, this illness is caused by severe acute respiratory syndrome coronavirus 2 (SARS-CoV-2), which is primarily spread from person to person [3, 4]. While some patients remain asymptomatic or have mild illnesses such as fever and cough, others develop severe syndromes, such as

5 Department of Medical Laboratory Sciences, College of Applied Medical Sciences, Majmaah University, Majmaah 11952, Kingdom of Saudi Arabia

6 Department of Bioengineering, Faculty of Chemical-Metallurgical Engineering, Yildiz Technical University, 34220 Istanbul, Turkey

7 Department of Mechanical Engineering, University of British Columbia, Vancouver, BC V6T 1Z4, Canada

8 Centre for Blood Research, Life Sciences Centre, University of British Columbia, Vancouver, BC V6T 1Z3, Canada 
pneumonia and acute respiratory distress syndrome, which may cause death [5]. As there are currently no specific vaccines or treatments for COVID-19, early and rapid diagnosis is crucial for preventing severe complications [6]. Prompt diagnosis is also critical for contact tracing and implementation of measures against further spread of COVID-19 [7, 8].

Various laboratory diagnostic tests have been broadly used in the current pandemic such as quantitative real-time polymerase chain reaction (qPCR), serology test, and chest computed tomography (CT) scan [9] (Table 1). To date, qPCR has been routinely used to detect virus-specific genes. While the test provides high sensitivity and specificity, it is labor-intensive and time-consuming as it can take up to 2 days to complete [18]. The serology test measures the host response against COVID-19 infection through detecting antibodies generated by the host immune system. However, it can take up to 2 to 3 weeks to develop a detectable level of antibodies and the target antibodies may also be detected in patients recovered from COVID-19 [19]. In fact, although serology test is much simpler and faster than qPCR, it appears to be more useful in confirming past viral exposure or assessing the true extent of the outbreak. Chest CT scan has also been used to diagnose symptomatic patients with suspected COVID-19 infection, especially those with respiratory failure or acute respiratory distress syndrome. However, the chest CT often appears normal in patients with mild or early infection [20]. Additionally, the radiological findings on chest $\mathrm{CT}$ can mimic other viral pneumonia, limiting its use in reliably ruling out COVID19 infections. While these conventional laboratory tests have been used to some extent for COVID-19, they are tedious, highly dependent on skilled personnel, and unavailable in resource-limited settings where COVID-19 outbreak may occur.
Significant efforts have been devoted to the development of point-of-care (POC) biosensors, such as chip-based and paper-based biosensors to substitute the conventional assays for the diagnosis of infectious diseases at the POC [21-23]. Due to the high demand for rapid testing to control the current pandemic, POC biosensors which are of low cost and easy to use and have sample-in-answer-out capability are highly desirable $[24,25]$. These biosensors are based either on the detection of nucleic acids or proteins from the respiratory samples (e.g., throat swab, sputum) or blood samples. They facilitate testing outside of laboratory settings or in developing countries where highly trained personnel and high-end infrastructures may not be available $[21,26]$. While these tests are desirable to combat the pandemic, multiple challenges are yet to be addressed to bring the technologies to clinical settings. The ideal POC biosensor should meet the ASSURED criteria, Affordable, Sensitive, Specific, User-friendly, Rapid and Robust, Equipment-free, and Deliverable to end users, and allow prompt decision-making to mitigate the transmission of COVID-19 [27-29].

This comprehensive review article focuses on the most recent advances in POC diagnostic devices for potential diagnosis of COVID-19 that meet ASSURED criteria, which is distinct from previous review papers where nanomaterial-based biosensors [30-33] and non-POC diagnostic techniques [34-44] were summarized. In addition, the commercial POC biosensors have not been critically reviewed [45-48]. In this review, the development of POC biosensors for the detection of proteins and viral nucleic acids for COVID-19 is comprehensively reviewed based on three main categories: chip-based biosensors, paper-based biosensors, and other biosensors, providing a comprehensive survey with regard to their usability and performance. We first discuss the significance of an early and prompt diagnosis of COVID-19 and pathogenesis of COVID-19 along with the key diagnostic

Table 1 Diagnostic tests for COVID-19

\begin{tabular}{|c|c|c|c|c|c|c|c|c|}
\hline Diagnostic test & Method & Diagnostic biomarker & Equipment required & $\begin{array}{l}\text { Assay } \\
\text { time }\end{array}$ & $\begin{array}{l}\text { Clinical } \\
\text { sensitivity } \\
(\%)\end{array}$ & $\begin{array}{l}\text { Clinical } \\
\text { specificity } \\
(\%)\end{array}$ & $\begin{array}{l}\text { Expertise } \\
\text { required }\end{array}$ & References \\
\hline CT scan & Chest imaging & - & CT scanner & $1 \mathrm{~h}$ & 97 & 25 & Yes & {$[10]$} \\
\hline \multirow[t]{2}{*}{ Nucleic acid detection } & qPCR & RdRP gene & qPCR machine & $4 \mathrm{~h}$ & 71 & - & Yes & [11] \\
\hline & LAMP & ORF1ab gene & PCR thermocycler & $20-30 \mathrm{~min}$ & 97.6 & NA & Yes & {$[12]$} \\
\hline \multirow[t]{5}{*}{$\begin{array}{l}\text { Antigen and } \\
\text { antibody detection }\end{array}$} & ELISA & Total Ab & $\begin{array}{l}\text { Fluorescent plate } \\
\text { reader machine }\end{array}$ & $\sim 2 \mathrm{~h}$ & 94.8 & 100 & Yes & {$[13]$} \\
\hline & ELISA & $\operatorname{IgG}$ and $\operatorname{IgM}$ & $\begin{array}{l}\text { Fluorescent plate r } \\
\text { eader machine }\end{array}$ & $\sim 2 \mathrm{~h}$ & 83.7 & 100 & Yes & [14] \\
\hline & LFA & IgG and IgM & - & $<15 \min$ & 88.6 & 90.6 & No & {$[15]$} \\
\hline & LFA & $\operatorname{IgM}$ & - & $<15$ min & 78.6 & NA & No & {$[16]$} \\
\hline & LFA & $\mathrm{IgG}$ & - & $<15 \min$ & 96.8 & NA & No & [17] \\
\hline
\end{tabular}

Abbreviations: CT: chest computerized tomography, Ab: antibody, ELISA: enzyme-linked immunosorbent assay, LFA: lateral flow assay. LAMP: loopmediated isothermal amplification 
biomarkers. Subsequently, we critically review the most recent advances in POC biosensors such as chip-based, paper-based, and other biosensors (e.g., textile-based and nanomaterial-based biosensors), which show tremendous potential for the detection of COVID-19. We highlight the benefits of each biosensor along with their use for the detection of main target analytes such as antigen, antibody, and nucleic acids. We subsequently review the commercial POC biosensors for COVID-19. Finally, the key challenges and future directions of biosensing research for COVID-19 are briefly discussed. This review would be very helpful in raising awareness about the importance of early and rapid diagnosis of COVID-19 and providing guidelines for developing ideal POC biosensors to manage the current outbreak.

\section{The importance of early and rapid diagnosis of COVID-19}

Generally, early and prompt diagnosis is critical to reduce the risk of developing serious complications. Severe COVID-19 infections are usually associated with risk factors such as age and immune status which could be life-threatening. For instance, elderly and immunocompromised patients are more likely to develop severe health issues. Specifically, people at the high risk of severe illness from COVID-19 include those who are taking immunosuppressant medicine or are undergoing cancer therapy. Meanwhile, people at the moderate risk of complications include the elderly (age $>65$ ) or those who have underlying health diseases such as asthma, diabetes, and kidney disease. Therefore, timely medical treatment is crucial to reduce the rate of disease progression and to prevent death [1].

Direct human-to-human transmission is the primary route of SARS-CoV-2 transmission and symptomatic people are the most frequent source of COVID-19 spread. Similar to other respiratory pathogens, the virus transmission generally occurs through respiratory droplets from coughing and sneezing. Aerosol transmission is also possible especially when one is exposed to large amounts of viruses in enclosed public spaces. Asymptomatic individuals may contribute to about $80 \%$ of the transmission [49]. The spread is primarily caused by family members, friends, co-workers, or close contacts. Contracting the virus present on the surfaces or objects may be another source of infection. However, the frequency of this type of transmission remains unclear [50]. The incubation period is generally within 3 to 7 days and symptoms may develop within 2 weeks following exposure to the virus [51]. Identification of diagnostic biomarkers enables accurate detection of SARS$\mathrm{CoV}-2$ infection as they can be used to determine the progress of infection. There are two main stages of infection: (i) the first or early stage of infection which happens when the patient is infected and (ii) the second stage of infection when the production of antibodies begins (Fig. 1) [52].

After exposure to the virus, the virus enters the lungs through the respiratory tract and attacks alveolar epithelial type 2 (AT2) cells [53]. At the early stage of infection, the viral RNA and the antigen produced can be readily detected.
Fig. 1 Major diagnostic markers for COVID-19. Following the infection, the number of SARSCoV-2 RNA increases dramatically at the early stage of infections, followed by an increase in the level of antibodies. Adapted with permission from [52]

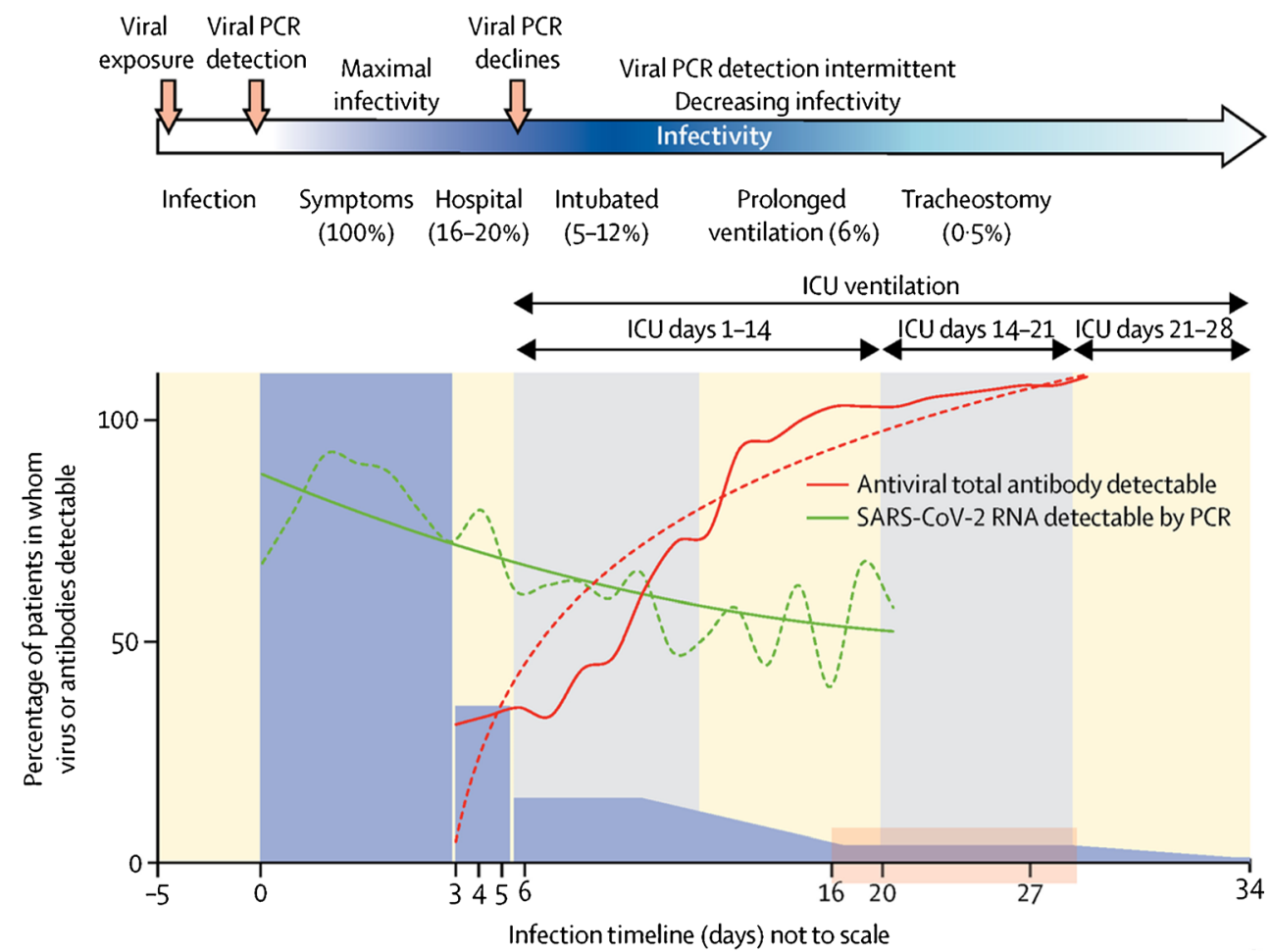


The rapid viral replication triggers a series of inflammatory reactions (e.g., fever, cough, and shortness of breath). Although most cases are mild, around $20 \%$ of cases become severe or critical with acute respiratory distress syndrome, multi-organ dysfunction syndrome, or sepsis, which are more likely to be life-threatening. The aggressive inflammatory response is usually accompanied with the exaggerated cytokine release or cytokine storm. An elevated amount of proinflammatory cytokines, such as interleukin-1 (IL-1), IL-6, and tumor necrosis factor-alpha (TNF- $\alpha$ ), could be found in severe and critical COVID-19 patients. Immunological abnormalities in COVID-19 patients include lymphopenia (reduction in numbers of $\mathrm{CD}^{+} \mathrm{T}$ cells, $\mathrm{CD} 8^{+} \mathrm{T}$ cells, natural killer (NK) cells, and B cells) and neutrophilia (increase in numbers of neutrophils) [54].

An increase in antigen level triggers the activation of $\mathrm{T}$ cells which stimulates the B cells to produce antibodies. The antibody responses against SARS-CoV-2 are mainly comprised of $\operatorname{IgM}$ and $\operatorname{IgG}$. Between day 7 and day 14 after the onset of symptoms, the antibody levels persist. In fact, while these antibodies are valuable serological markers of infection [55], their role in controlling viral replication has not been well defined [56]. The nature of immune cell long-term memory responses to the virus also remains unknown. As COVID19 shows a large range of clinical manifestations (from mild symptoms to life-threatening conditions), rapid tests are essential especially during the early stages of infection to identify positive cases.

Current laboratory tests such as qPCR, chest CT, and serology tests are commonly performed to detect the broad spectrum of COVID-19 clinical presentations. qPCR is known as a gold standard for SARS-CoV-2 detection, and has been recommended by WHO, which has high sensitivity and specificity [57]. During the febrile/viremic phase (i.e., the first 3-5 days following the onset of symptoms), the sample is usually collected from the lower respiratory tract for RNA isolation [58]. However, this test is expensive and time-consuming (i.e., almost a week) and requires highly skilled laboratory personnel [35]. To shorten assay time, reverse transcription loopmediated isothermal amplification (RT-LAMP) has been used for COVID-19 detection [59]. This test is performed in an isothermal condition, and the results are obtained within 15$40 \mathrm{~min}$, by targeting the genes of SARS-CoV-2 [60, 61]. The RT-LAMP result can be evaluated using real-time electrophoresis, turbidimeter, or fluorescent, which is faster, cheaper, and more convenient than qPCR [58]. Serology tests such as IgG or IgM-based enzyme-linked immunosorbent assay (ELISA) kits are the cheapest and simplest tests, which have been broadly used for the diagnosis of COVID-19. However, patients with past infection are tested positive as IgM remains in the body for weeks or months. In addition, a false-positive result usually appears in patients with other infections (e.g., influenza) due to the possibility of cross-reactivity.
On the other hand, CT scan appears to be useful in detecting patients with COVID-19 pneumonia [62]. The chest CT scan combines images from various angles of the chest and produces cross-sectional lung images through image processing to diagnose pneumonia. However, the screening usually requires complex benchtop medical instruments, which are only available in healthcare facilities or hospitals [63]. In addition, CT scan might be less accurate to diagnose COVID-19 as the result appears normal in asymptomatic and mildly symptomatic patients without pneumonia [38]. Taken together, while conventional laboratory tests could successfully diagnose COVID-19 infections, they are unable to fulfill the criteria for a POC test (e.g., rapidity, portability, and affordability) at resource-limited settings or developing countries. Therefore, there is an urgent need to develop low-cost and simple biosensors for COVID-19 at the POC.

\section{Recent advances in POC technologies for COVID-19}

\section{Fundamental of POC biosensors}

Biosensors are analytical devices used for the detection of a biological substance. In general, biosensors consist of three components: a receptor (specific for a disease) that recognizes the analyte, a transducer that converts the bio-recognition event into a measurable signal, and a reader [64]. This technology allows precise control and manipulation of fluids, which typically requires much less sample volume than that of conventional assays [24]. The efficient liquid mixing in biosensors also enhances the interaction between assay reagents and target biomarkers, which shortens the assay duration and provides fast readout $[65,66]$. Moreover, the portability of biosensors makes them ideal candidates for POC field settings. To date, researchers around the globe have developed various types of biosensors for wide applications in POC settings such as the diagnosis of infectious diseases, food safety analysis, and environmental monitoring [67-69]. These biosensors include chip-based, paper-based, and other biosensors (textile-based or nanomaterial-based biosensors), which will be briefly discussed in the following sections.

For POC testing of chronic and infectious diseases, there is always an increasing demand for low-cost, portable, and integrated biosensors, which can provide rapid results with low sample consumption. Chip-based biosensors are one of the POC biosensors used for POC diagnosis of many infectious diseases. They are prepared either by miniaturizing conventional biochemical assays on a microchip scale $[70,71]$ or by integrating novel detection principles with microfluidic chips $[72,73]$. These biosensors are mainly made of polymethyl methacrylate (PMMA), polytetrafluoroethylene (PTEE), or polydimethylsiloxane (PDMS) [74, 75]. PDMS biosensors 
are commonly used due to their cost-effectiveness, high specificity, and minimal reagent consumption [76]. Specifically, they consist of multiple channels which enable nucleic acid testing steps, including nucleic acid extraction, amplification, and amplicon detection, to be performed in an automated manner [24]. In recent years, smartphone has also been integrated into chip-based biosensors for imaging and signal analysis. For instance, chip-based biosensors coupled with a smartphone have been used to rapidly detect amplicon signals within an hour for the diagnosis of H1N1 [77] and Zika virus infections [78].

Paper-based biosensors have been broadly used for rapid testing of infectious diseases, which show potential to substitute the conventional laboratory tests and chip-based biosensors $[79,80]$. Paper is inexpensive, readily available, and biodegradable, showing a promising tool for onsite rapid diagnosis [21]. It allows the diffusion of a biological sample through a capillary effect, eliminating the need for external power sources. Earlier studies have introduced lateral flow test strips and microfluidic paper-based analytical devices $(\mu \mathrm{PAD})$ for POC testing [22, 23]. The assay usually involves hybridization of single-stranded DNA or RNA with a complementary probe to produce double-stranded nucleic acids or interaction between antigen and antibody to produce an Ag-Ab complex, generating signals such as colorimetric, fluorescence, or chemiluminescence signals [81]. Their special characteristics such as simple, affordable, and ease of fabrication, modification, and functionalization have made them possible to achieve rapid, onsite POC testing.

Other biosensors like film-based, textile-based, and nanomaterial-based biosensors have also been used for the diagnosis of infectious diseases. For instance, film-based biosensors which are made of transparent polyester substrate film have been used for the detection of pathogens. This material has the ability to withstand thermal cycling and amplification process $[82,83]$. Textile-based biosensors are biosensors which are typically made of thread, fabric, or clothes which are inexpensive and readily available with low sample consumption. For example, textile-based biosensors are cheaper and require a smaller sample volume $(<\$ 1$ USD/assay, $\sim 20$ $\mu \mathrm{L}$ sample) compared to lateral flow test strip (\$3 USD/assay, $\sim 50 \mu \mathrm{L}$ sample) [84]. In addition, nanomaterial-based biosensors which are made of nanomaterials such as graphene or graphene oxide have been reported to have higher sensitivity compared to other biosensors $[85,86]$. These emerging biosensors offer tremendous potential to substitute chip-based and paper-based biosensors for rapid testing at the POC.

\section{POC biosensors for the detection of antigens and antibodies for COVID-19}

As mentioned earlier, one of the most common tests for COVID-19 is the serology test (i.e., ELISA), which specifically detects IgG/IgM, viral antigens, or cytokines $[87,88]$. Nevertheless, this test suffers from tedious assay protocols and fails to provide rapid feedback to healthcare professionals. Furthermore, a benchtop microplate reader/ scanner is often required for quantitative readouts. In fact, in response to the current pandemic, periodic testing of patient samples is required for efficient disease diagnosis and management [89]. Therefore, there is a high demand for rapid, cost-effective, and easy-to-use detection approaches to produce a timely result for responsive COVID-19 monitoring. All of these demands call for the development of POC biosensors for fast and accurate detection of COVID-19 specific biomarkers at the POC.

\section{Chip-based POC biosensors for the detection of antigens and antibodies}

To date, conventional fluorescence and colorimetric assays have successfully transformed from multi-well plates into chip-based biosensors, which allows rapid and accurate COVID-19 diagnosis. For instance, Joh et al. [83] have developed an on-chip fluorescence-based ELISA in a more compact footprint that can be operated without any fluidic setup (Fig. 2a). The vacuum-sealed chips can be stored for up to 92 days under ambient environment or 5 days at $45^{\circ} \mathrm{C}$ without losing noticeable performance. Facing the present COVID-19 crisis, this format of the biosensor (on-chip ELISA test) has seen immediate translation for rapid and portable serological detection of COVID-19 [91, 93]. Liu et al. [85] incorporated the centrifugal microfluidics technique with a fluorescent immunoassay for detecting $\operatorname{IgG}, \operatorname{IgM}$, and viral antigen of SARS-CoV-2. Each analyte is selectively detected on an individual microfluidic chip, while they are simultaneously analyzed in a portable device that combines liquid handling and signal readout for POC diagnosis (Fig. 2b). The built-in optical unit illuminates the detection zone with a laser of desired excitation wavelength and measures the intensity of emitted fluorescence signal. The entire process takes only $15 \mathrm{~min}$ from sample loading to signal readout, serving as an ideal POC detection platform for the fast-evolving COVID-19 pandemic. In particular, the simultaneous analysis of viral antigen and corresponding antibodies allows a specific and accurate diagnosis of SARS-CoV-2, overcoming the transient expression of IgM in the blood [94] and the cross-reaction of antibodies targeting multiple strains of coronaviruses [95, 96]. This methodology could readily incorporate more target molecules for a multiplexed COVID-19 test to provide a robust, selective, and fast diagnosis.

Other alternative detection approaches have been introduced to provide quantitative and fast test results. Electrochemical biosensors can sensitively detect the electrical signals induced by biochemical reactions. Photonic biosensors can transduce the molecular binding events to optical 
a
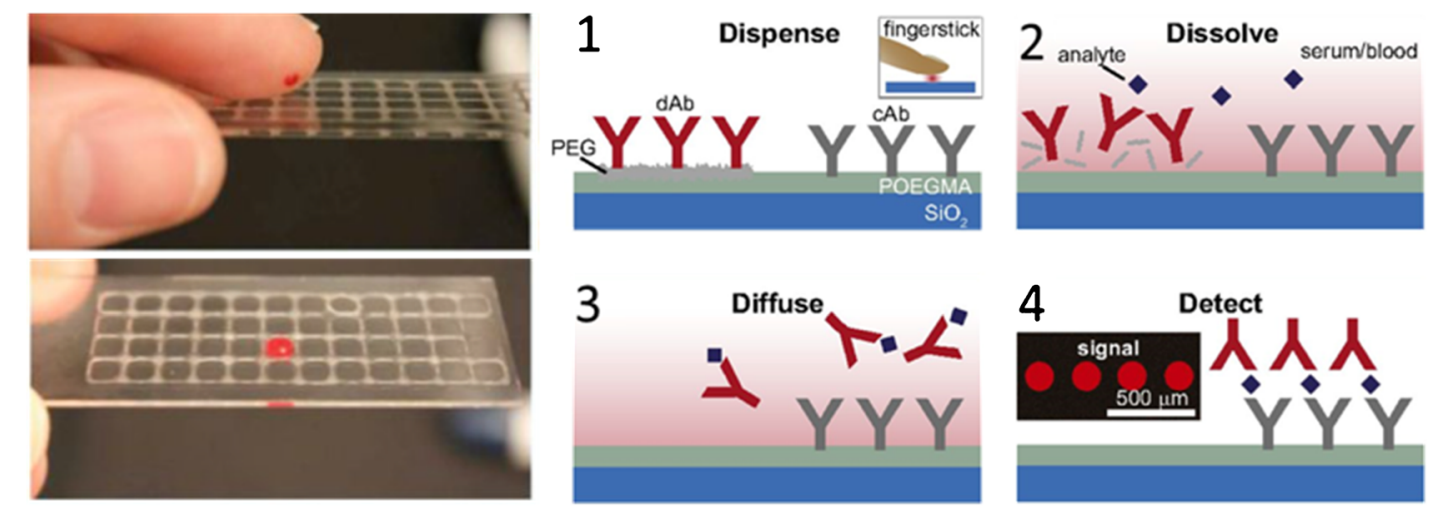

b

C
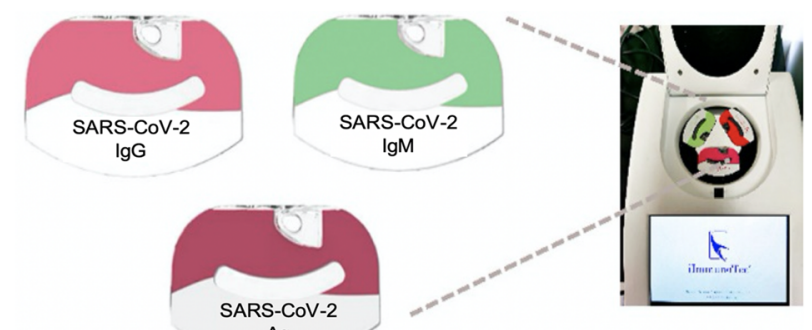

$\mathrm{Ag}$
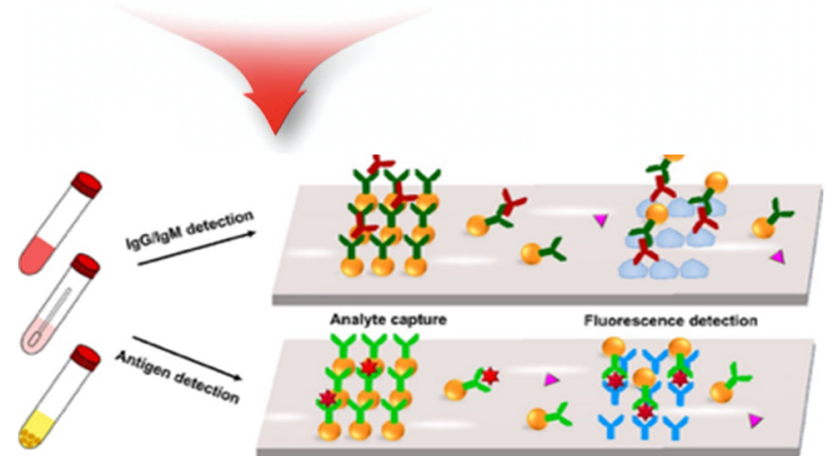

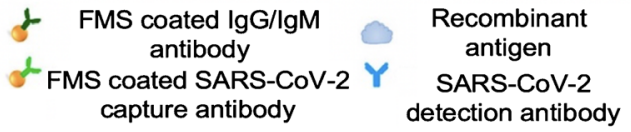

$Y \lg G / \lg M$

Antigen capture antibody

detection antibody
Fig. 2 Chip-based biosensors for potential antigen and antibody detection for COVID-19. a Miniaturized and high-throughput ELISA on a chip-based biosensor. The fingerstick blood is directly applied to an assay chamber. The slide is prepared by printing "stable" spots of capture antibodies (cAb) and "soluble" spots of the fluorescently tagged detection antibodies (dAb) on the polymer brush (1). dAb dissolves and binds to the analyte (2), the complexes diffuse and bind to the respective cAb spots (3), and subsequently (4) generates fluorescent signals. A

signals. Therefore, extensive studies have exploited these biosensors for POC diagnoses, especially by integrating with mobile phones [97]. For instance, Sun et al. [90] prototyped a miniaturized printed circuit board (PCB) module for mobile phone-based POC detections (Fig. 2c). Using the welladopted sandwich ELISA format, this portable module can detect a wide range of clinically relevant concentrations of secretory leukocyte protease inhibitor (SLPI), an established mobile phone-based reader is coupled with the biosensor to provide quantitative readout. Adapted with permission from [90]. b Multiplexed detection of $\operatorname{IgG}, \operatorname{IgM}$, and the viral antigen for SARS-CoV-2 based on fluorescence immunoassay using a centrifugal chip-based biosensor (FMS: fluorescent microsphere). Adapted with permission from [91]. c A low-consumption electrochemical biosensor for antigen detection, which is powered directly by a mobile phone. Adapted with permission from [92]

biomarker for lung infections. The detection results can be immediately interpreted and saved by the phone and communicated remotely with medical cloud service or doctors for further validations. Compared to the electrochemical mechanism, the photonic biosensors can achieve a high signal-tonoise ratio $[98,99]$. Several studies have demonstrated the applications of photonics techniques for POC detection and diagnosis [100, 101]. Belushkin et al. [72] investigated the 
early diagnosis of sepsis by using a compact photonic detection setup. The device can reach an excellent detection limit of $36 \mathrm{pg} / \mathrm{mL}$ for C-reactive protein (CRP) and $21 \mathrm{pg} / \mathrm{mL}$ for procalcitonin $(\mathrm{PCT})$, enabling the identification of healthy controls against sepsis and noninfectious systemic inflammatory response syndrome (ni-SIRS) patients by using clinical human serum samples. More importantly, the signal output takes less than 15 min, outrunning the current gold standard laboratory tests. The device packaged in a compact portable assembly shows a bright potential for performing fast and accurate cytokine assays for COVID-19 in clinics and POC settings for responsive disease management.

\section{Paper-based POC biosensors for the detection of antigens and antibodies}

Besides chip-based biosensors, paper-based biosensors such as lateral flow test strips and $\mu \mathrm{PAD}$ have emerged to provide a cost-effective and rapid solution to POC needs. As a versatile detection tool, paper-based biosensors have been widely used in medical diagnostics for home/POC testing and laboratory uses. Recent studies have exploited this assay format for detecting infections [102, 103], cancer biomarkers [104], and other diseases $[105,106]$ based on antigens or antibodies, which can potentially be used for COVID-19. For instance, Brangel et al. [102] introduced a paper-based serology test for POC detection of ebola virus infections (Fig. 3a). The test eventually obtained highly comparable detection performance to the standard ELISA concerning sensitivity (100\%) and specificity (98\%). Furthermore, the authors developed a customized mobile phone application for semi-quantifying the test results (colorimetric intensity-based) and recording the patient data (such as the test history), representing a systemic method for epidemic surveillance and patient management.

By adopting a similar detection strategy, Li et al. [103] developed a quick paper-based serology test for the current COVID-19 pandemic. This assay targets the two human immunoglobulin isotopes, namely IgG and IgM, that are known to be involved in SARS-COV-2 infections [108](Fig. 3b). To evaluate the test sensitivity and specificity, 525 patient blood samples were collected, of which 397 were clinically confirmed positive and 128 were negative. Each test consumes 15-20 uL of blood and took around $15 \mathrm{~min}$, achieving a sensitivity of $88.66 \%$ and specificity of $90.63 \%$. In particular, 12 clinically negative samples were diagnosed positive (thus false-positive) with the paper strip. This outcome may not qualify the proposed test for robust clinical diagnosis. On the one hand, the authors speculate that the early onset and rapid denaturation of IgM [94] in blood samples would generate false-negative signals hence result in lower test sensitivity. On the other hand, the potential structural homology for Spike protein binding between COVID-19 antibodies and those against other coronaviruses $[95,96]$ would create false-positive results. Therefore, continuous in-depth virological studies are in urgent need to discover COVID-19 highly specific antigens for developing more accurate POC tests.

Recent endeavors have shown successful integration of microfluidics technique with paper-based detection approach to create $\mu \mathrm{PAD}$, which have improved fluidic control (e.g., mixing, merge, and delay) and enhanced test performance [109]. For instance, Tenda et al. [107] achieved the simultaneous testing of three anti-viral antibodies (anti-HIV1, antiHA (hemagglutinin), and anti-DENV1 (Dengue Virus Type I)) in whole blood using a 3D- $\mu \mathrm{PAD}$. The device is assembled vertically, where a plasma separation membrane is preinserted between the sample-loading region and the detection pad, obviating the blood separation steps and ensuring a simple test operation (Fig. 3c). Indeed, the entire test only requires a single step of sample addition (e.g., $20 \mu \mathrm{L}$ blood) without any buffer washing steps, followed by $20 \mathrm{~min}$ of incubation and a final image acquisition. The integration of paper-based detection and microfluidics enables processing of complex samples (e.g., serum and whole blood) and multiplexed POC assays for identifying multiple disease biomarkers, which holds great potential for identifying epidemic infection and monitoring the progressive cytokine storm in COVID-19.

\section{Other POC biosensors for the detection of antigens and antibodies}

Besides chip-/paper-based biosensors, various biosensors have been developed to detect biomarkers in a sensitive and specific manner, including those which are based on nanomaterials (e.g., nanowires, nanotubes, and nanoparticles) $[110,111]$ and 2D materials (e.g., graphene and graphene oxide) $[86,112]$. They have shown promising applications for POC detections. For example, Chen et al. [113] exploited the bioluminescence phenomenon found in fireflies for ultrasensitive antigen detection with nanomaterials-in-a-tube (Fig. 4a). Specifically, the authors adopted a strategy of sandwiched immunoassay where cAbs are bound on magnetic nanoparticles (termed as Ab1-MNPs), and dAbs are immobilized on polystyrene nanoparticles carrying the alkaline phosphatase (termed as Ab2-PS-ALP). The two nanoparticles anchor one identical antigen, generating an immune-nanocomplex of MNPs-Antigen-PS-ALP that can be enriched magnetically. Therefore, the number of ALP-presenting complexes is directly correlated to the abundance of antigens in the sample. By simply modifying the probes on the nanoparticles, this method could potentially enable ultrasensitive detection of COVID-19 viral antigens. Apart from the soluble nanoparticles, solidbased nanomaterials have also shown great sensitivity for biosensing. Nanowires made of semiconductor materials or metal oxides have reached a detection limit in the range of aM to $\mathrm{fM}$ for protein biomarkers [116, 117]. For example, Gao et al. [114] fabricated an array of silicon nanowires for a 
a
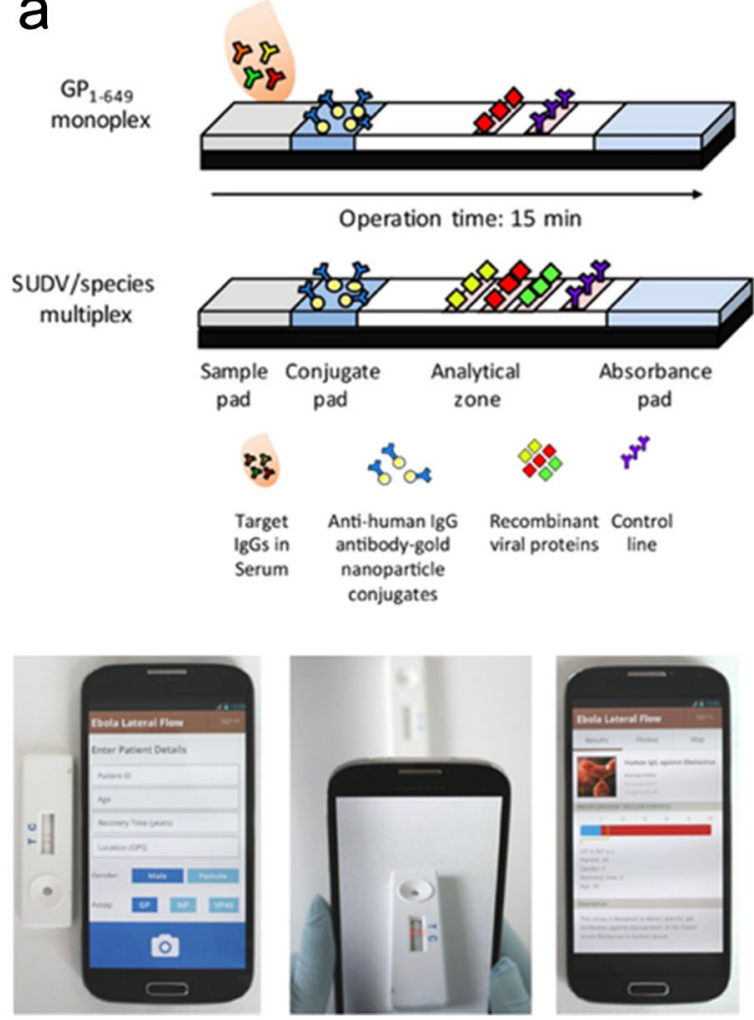

Operation time: $15 \mathrm{~min}$
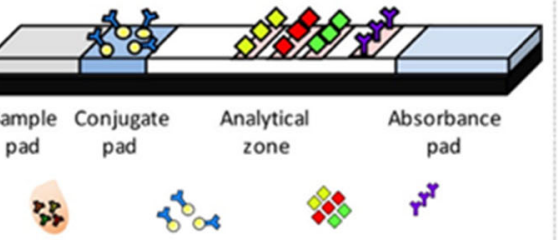

Target Anti-human $\lg G$

IgGs in antibody-gold

nanoparticle conjugates
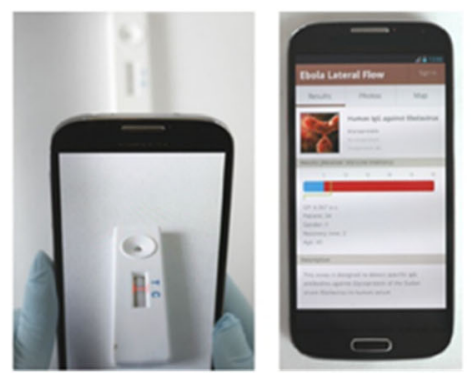

Fig. 3 Paper-based biosensors for potential antigen and antibody detection for COVID-19. a Monoplexed and multiplexed paper-based biosensors or lateral flow test strips for the detection of antibodies specific to the viruses. A customized phone application records the results along with the patient data for disease management. Adapted with permission

multiplexed electrochemical detection of lung cancer biomarkers: miRNA-126 and carcinoembryonic antigen (CEA). The capture probes were pre-functionalized on the nanowires ( $\sim 60 \mathrm{~nm}$ wide) (Fig. $4 \mathrm{~b})$ to achieve a real-time and label-free analysis. Notably, $10 \mathrm{fg} / \mathrm{mL}\left(10^{-15} \mathrm{~g} / \mathrm{mL}\right)$ of CEA can be reliably detected in serum samples, representing an ultrasensitive tool for early diagnosis of cancer, as well as other diseases with specific biomarkers, including COVID-19.

2D materials in the form of nanosheets or nanoparticles, such as graphene, graphene oxide, and black phosphorus, have drawn considerable attention in material science and biomedicine ever since the discovery of graphene in 2004 [118]. Numerous studies have explored their potential in biosensing and diagnostics, mainly by integration with existing electrodes or optical units for improving the device performance [119]. For instance, a black phosphorus-based biosensor was introduced for the detection of rabbit IgG [115] (Fig. 4c). Briefly, black phosphorus nanoparticles (BPNP) were synthesized by exfoliation of layered black phosphorus microparticles. These BPNP present an excellent electrocatalytic activity, which were utilized as electrocatalytic tags for the immunoassay. The paramagnetic beads (MB) were conjugated with antirabbit IgG to form MB-anti-rabbit IgG. In the presence of

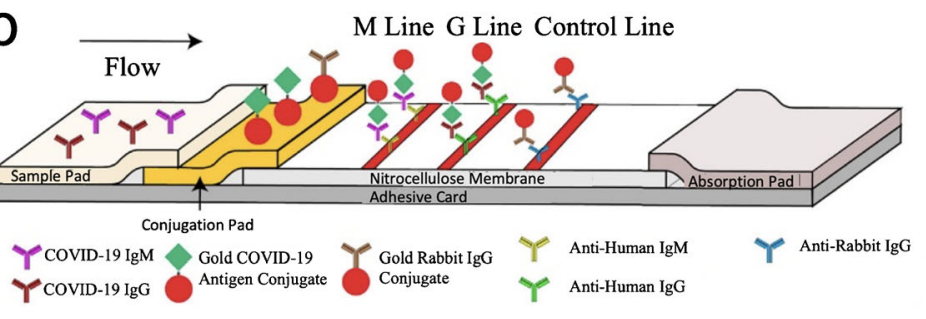

Hole in lamination
film $(d=5.5 \mathrm{~mm})$$\quad$ Thickness: $50 \mu \mathrm{m}$

Plasma separation

membrane $(d=7.9 \mathrm{~mm})$
pper paper layer impregnated

ower paper layer patterned

antibody detection $180 \mu \mathrm{m}$ ' Furimazine

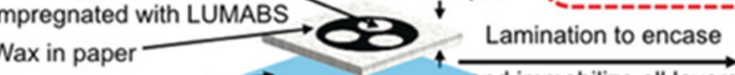

amination film $\longrightarrow \underset{\text { and immobilize all layers }}{\longrightarrow}$

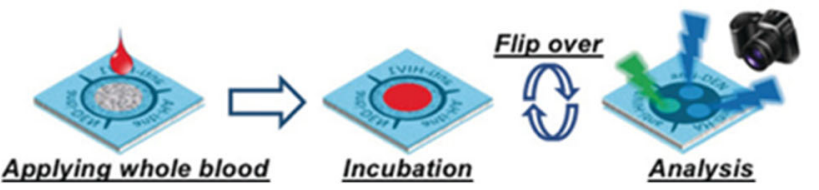

from [102]. b A paper-based biosensor which detects IgG and IgM for COVID-19. Adapted with permission from [103]. c 3D- $\mu$ PAD and its simple operation for simultaneous detection of three different antibodies for medical diagnosis. Adapted with permission from [107]

targets rabbit IgG, the targets bind to the BPNP to form complexes which in turn interact with MB-anti-rabbit IgG. The subsequent addition of sulfuric acid would result in complex denaturation, which would ultimately generate an electrochemical reaction on the electrode surface. The target IgG concentration was measured by electrochemical signal produced by the electrocatalytic activity. This BPNP-based biosensor was highly sensitive and specific, which shows great promise for human IgG detection for COVID-19.

Apart from the above biosensors that require dedicated nanotech facilities, others have been focusing on improving the existing biosensors (e.g., sensitivity and ease of use) with simple fabrication processes. Textile-based biosensors have been developed, among others, with wearable or portable features and improved assay performance [120, 121]. Moreover, Choi et al. [84] optimized the fluid transportation in a threadbased biosensor (a type of textile-based device) for sensitivity enhancement (Fig. 4d). The fluid flow is delayed by coating the thread with hydrophobic polysiloxanes, resulting in more efficient antibody-antigen interaction for the lateral flow assay. The detection sensitivity of bacteria S. Enteritidis increased by 10 -fold compared to non-modified thread, namely $500 \mathrm{CFU} / \mathrm{mL}$ versus $5000 \mathrm{CFU} / \mathrm{mL}$. This simple-to-fabricate 


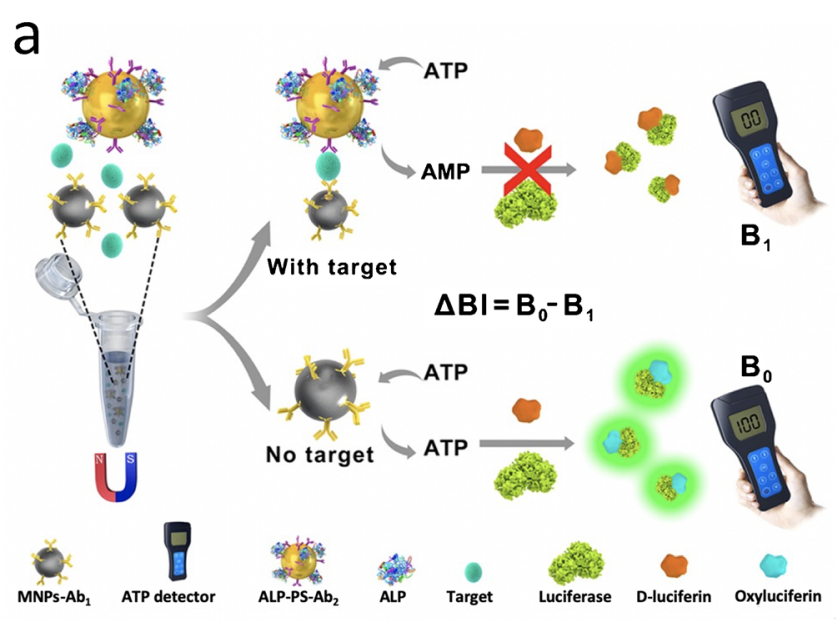

b

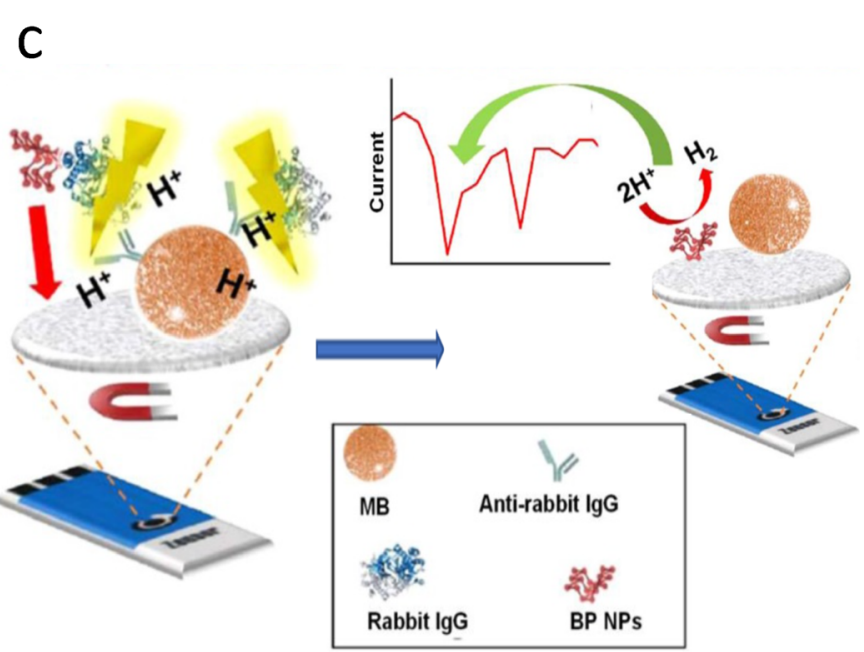

Fig. 4 Other point-of-care biosensors for potential antigen and antibody detection for COVID-19. a A nanoparticle-based portable bioluminescent immunosensor (ABS). Adapted with permission from [113]. b An array of silicon nanowire biosensor for sensitive detection of disease biomarkers. Adapted with permission from [114]. c

and sensitive biosensor shows immense potential in detecting IgG and IgM for a quick COVID-19 serology test. In the future, more investigations are required to ensure reproducible biosensor manufacture and consistent performance, and mobile readout methods will promote the biosensor industry and revolutionize the POC diagnostics.

\section{POC biosensors for the detection of nucleic acids for COVID-19}

The detection of viral nucleic acids at the early stage of COVID-19 infection presents a higher sensitivity and specificity compared to antigen or antibody detection [37]. In general, nucleic acid testing involves three main steps: nucleic acid extraction, amplification, and detection [122-124]. Most COVID-19 tests have been developed to detect the SARS-CoV-2 specific genes including the RNA-dependent
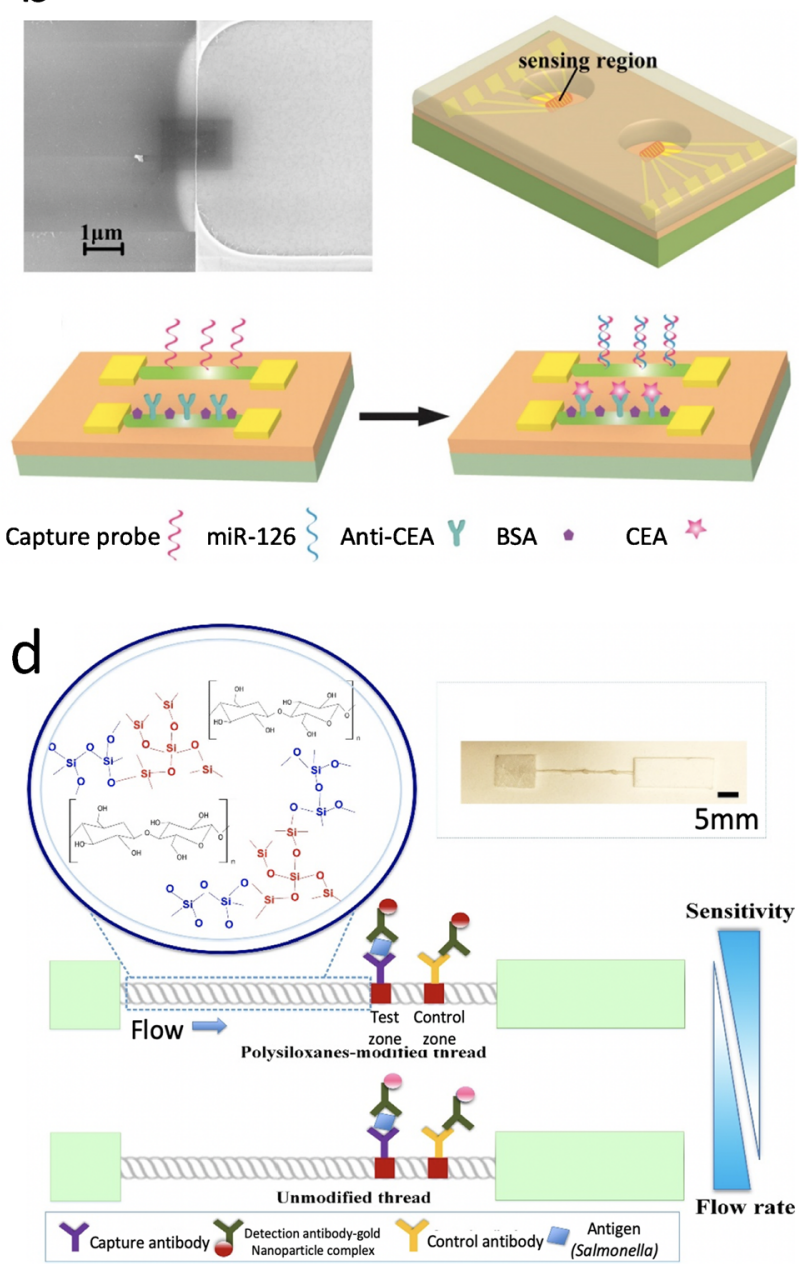

Capture probe $\{\operatorname{miR}-126\}$ Anti-CEA Y BSA * CEA $\$$

A black phosphorus-based biosensor for electrochemical detection of IgG. Adapted with permission from [115]. d A polysiloxanemodified thread-based biosensor for sensitive detection of pathogens. Adapted with permission from (196)

RNA polymerase (RdRP) gene, Nucleocapsid (N) gene, Envelope (E) protein gene, Spike protein (S) gene, and ORF1ab gene $[125,126]$. The conventional nucleic acid test typically requires time-consuming and complicated operation processes, which are not suitable for POC testing [127]. Therefore, there is an urgent demand to develop rapid, costeffective, and highly sensitive POC biosensors to manage the current pandemic [128].

\section{Chip-based POC biosensors for the detection of nucleic acids}

In general, most of the chip-based biosensors for nucleic acid testing are made of glass because of its transparency and low auto-fluorescence [129-131]. However, temperature distribution is not uniform due to the low thermal conductivity of glass. Since PDMS has advantages including biocompatibility, low cost, and simple processing, it has been widely utilized 
for the fabrication of miniaturized devices. While nucleic acid amplification can be performed in a disposable PDMS-glass chamber, the evaporation of reagents in the PDMS chamber may result in cross-contamination due to the porous nature of PDMS. In contrast, PMMA is currently used to fabricate chipbased biosensors via laser ablation due to its low rate of evaporation and low non-specific nucleic acid adsorption [132]. There are many types of chip-based POC biosensors for COVID-19 such as colorimetric, fluorescence, and electrochemical chip-based biosensors.

A colorimetric biosensor based on a LAMP reaction was developed to detect SARS-CoV-2 nucleic acids [133]. The 3D-printed incubation chamber connected to a water circulator was designed for sample incubation at $50-65^{\circ} \mathrm{C}$. SARS$\mathrm{CoV}-2$ genetic sequences were amplified for $30 \mathrm{~min}$. Due to the chemical reaction between DNA polymerases and a deoxynucleoside triphosphate during LAMP amplification, a hydrogen ion is produced as a byproduct that results in a color change from red to yellow in the presence of phenol red. Positive nasopharyngeal samples were able to be distinguished from negative samples with the use of CIELab space as a color analyzer in the range of 62 to $2 \times 10^{5}$ DNA copies. qPCR was used to validate the proposed biosensor and the results were compatible with each other. To allow more accurate quantification, Sun et al. [134] have developed a fluorescence chip-based biosensor integrated with a smartphone as a model diagnosis system for detection of COVID-19. The equine respiratory sample was used as a model system for the diagnosis of COVID-19. At first, primers were injected into the microfluidic channels and dried. Then, the sample was introduced to the channel, and evaporation was prevented with the use of a layer of double-sided adhesive and a cover glass. A heating step was performed at $65^{\circ} \mathrm{C}$ for the LAMP enzymatic amplification reaction. After LAMP amplification, fluorescence images were captured by a smartphone based on emission of the EvaGreen DNA-intercalating dye to obtain an average value of the pixel intensity for detection of target nucleic acids. With this biosensor, Equine herpesvirus 1 (EHV1) from horse nasal swab was detected in 30 min with a detection limit of 18 copies per reaction. The biosensor is promising for POC detection of COVID-19 with the advantages including rapidity, inexpensiveness, portability, robustness, and ability to perform multiplex detection at a time. Additionally, it allows simultaneous POC health diagnostic while reduces false-positive or negative results due to the existence of multiple channels on the chip. Another fluorescence chip-based biosensor based on LAMP was demonstrated by Ganguli et al. [135] for real-time detection of SARS-CoV-2. At first, Orf 1a, S, Orf 8, and $\mathrm{N}$ genes were identified and analyzed using BLAST for target primers. Then, RT-LAMP reaction was loaded into the 3D-printed microfluidic polymer cartridges that consist of a heater and optics via syringes. The RT-LAMP amplification was performed at $65^{\circ} \mathrm{C}$ using a self- regulating positive temperature coefficient heater. EvaGreen dye, a double-stranded DNA-intercalating dye, was used to generate emission light. Finally, the fluorescence signal from amplicons was recorded via a smartphone followed by analysis using ImageJ software. The detection limit of 5000 RNA copies/ $\mu \mathrm{L}$ in the nasal samples was obtained in less than 40 minutes with the use of a handheld reader at POC. For multiplexed detection of different pathogens, the first polymer/paper hybrid microfluidic biochip integrated with LAMP was reported by the Li group [136] (Fig. 5a). The biochip includes three layers; the top layer and middle layers are made of PDMS with a central inlet reservoir and 4 microchannels for reagent delivery, and 8 LAMP zones for detection, respectively. Different LAMP zones, which consist of laser-cut chromatography paper disk, were used for simultaneous detection of various pathogens including $N$. meningitidis, S. pneumoniae, and Hib within $1 \mathrm{~h}$. A glass slide was utilized as a structural support at the bottom part of the chip. After commercial LAMP mixture was prepared and added to the LAMP zones on chip-based biosensor through the inlet, epoxy glue was used to cover the inlet and outlets. Next, a low-cost portable heater designed by the group was used to heat the microfluidic chip for LAMP reactions. It is possible to directly analyze samples without the necessity of sample preparation steps owing to the integration of centrifuge-free lysis step on the chip. Fluorescence images were captured by a cellphone camera under a portable UV light pen after LAMP reactions occurred. The detection limit of $N$. meningitidis, S. pneumoniae, and Hib were obtained as 3 copies, 6 copies, and 5 copies per LAMP zone, respectively. This biosensor was further improved by the same group and applied for the detection of whooping cough (pertussis) [137] (Fig. 5b). The difference from the previous biosensors is that they developed a home-made battery-powered heater in addition to a 3D-printed integrated bioanalyzer for fully POC diagnosis. The detection limit of 5 DNA copies per LAMP zone was obtained. The ready-to-use hybrid biosensor offers inexpensive detection with a cost per assay of $\sim 3$ dollars. Also, this biochip provides a fully battery-powered system without the need for an external power supply, which can be easily adapted for POC detection of COVID-19.

Similarly, a smartphone has also been coupled with a chipbased electrochemical biosensor for the detection of SARSCoV-2 [139]. This biosensor is made of ion-sensitive fieldeffect transistors (ISFETs) using complementary metal-oxidesemiconductor (CMOS) technology. After the sample reaction mixture was placed into a disposable 3-D printed cartridge consisting of a CMOS ISFET microchip (4368 sensors array), a sensing experiment was conducted in a portable chip-based biosensor. This biosensor includes an $\mathrm{Ag} / \mathrm{AgCl}$ reference electrode and a manifold consisting of two microfluidic channels for the sample and control zone. The nucleic acid extraction and amplification (LAMP) steps were performed in a tube 
a

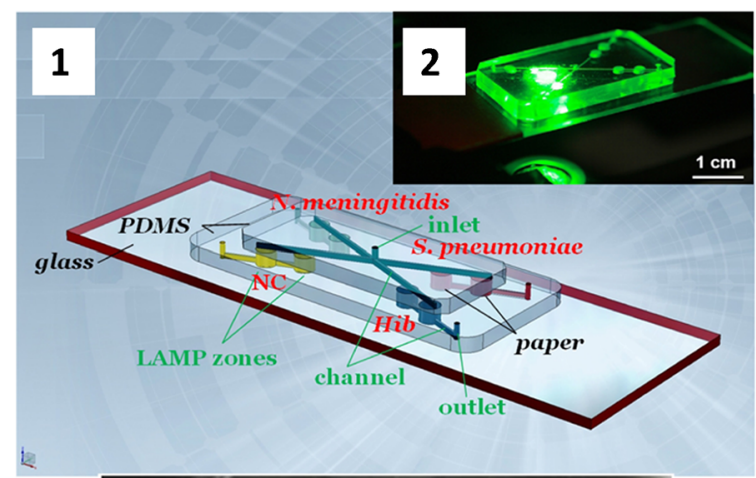

3

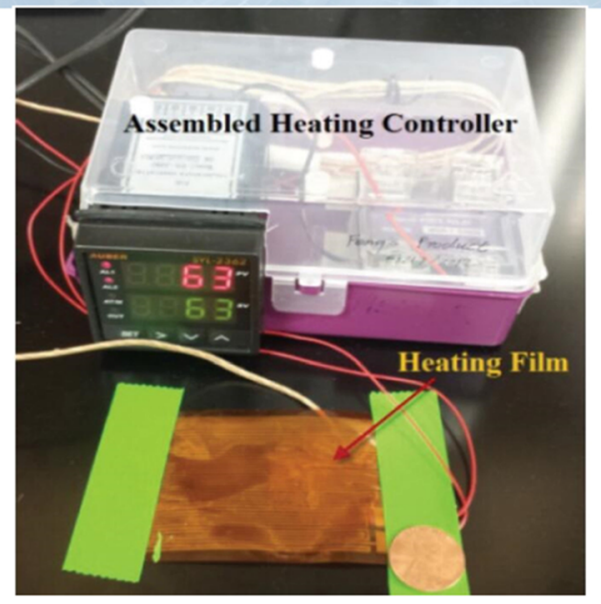

Fig. 5 Chip-based biosensors for potential nucleic acid detection for COVID-19. a A PDMS/paper hybrid chip-based biosensor for pathogen detection. Adapted with permission from [136]. b A chip-based biosensor integrated with loop-mediated isothermal amplification (LAMP) and

(off-chip), and the amplicons were added into the developed biosensor for nucleic acid detection. Patient clinical samples such as nasopharyngeal, throat specimens, and nose swabs were used to validate the biosensor with a detection time of $<20 \mathrm{~min}$. The voltage change due to the $\mathrm{pH}$ variations was recorded and analyzed using a custom smartphone application developed on AndroidOS. The diagnosis platform had a detection limit of 10 copies of DNA per reaction with sensitivity and specificity of $90.55 \%$ and $100 \%$, respectively. The developed biosensor is advantageous over lateral flow test based on CRISPR-Cas12 [140] due to its ability to provide quantitative results. The biosensor provides portable, low-cost, and rapid quantification of SARS-CoV-2 RNA along with a smartphone application. Another ISFET biosensor using a CMOS Lab-onChip platform was presented for Plasmodium falciparum malaria diagnosis by Malpartida-Cardenas et al. [138] (Fig. 5c). The biosensor was fabricated using unmodified CMOS technology and LAMP. DNA amplification detection was performed in a microfluidic chamber made of acrylic sheet and integrated on top of the CMOS chip. After pH-LAMP reagents were loaded to the microfluidic chamber, DNA b

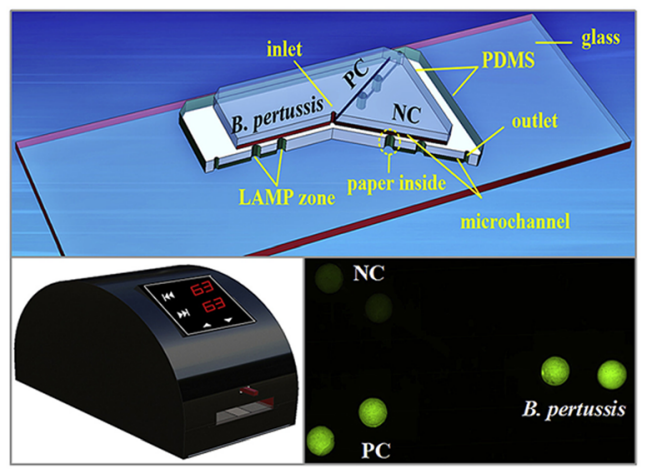

C

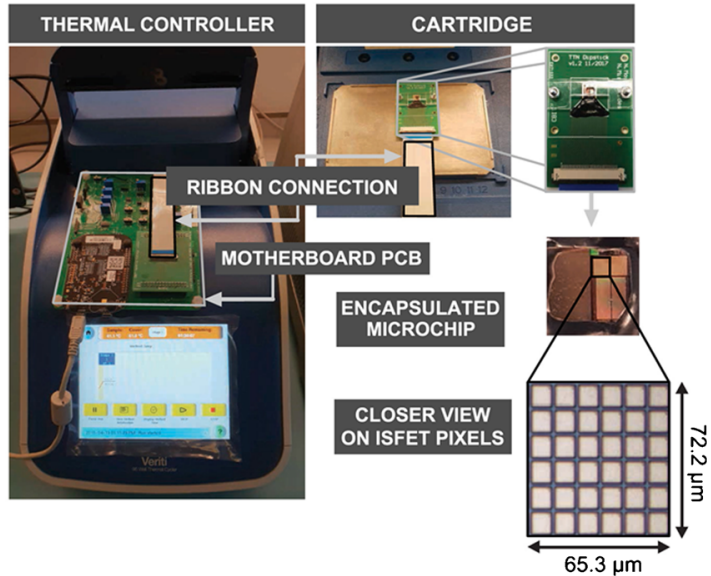

portable battery-powered heater. Adapted with permission from [137]. $\mathbf{c}$ A lab-on-a-chip platform which consists of an array of ISFET chip-based biosensors coupled with a thermal controller. Adapted with permission from [138]

amplification reactions occurred. A printed circuit board (PCB) was utilized to control temperature during amplification. A proportional electronic (voltage) change was obtained due to a change in $\mathrm{pH}$ of the loaded solution as a result of DNA amplification followed by sensing via ISFETs. The analysis time was less than 20 min. Real-time data acquisition was achieved by a Matlab-based graphical user interface. Although the proposed method is promising for sensitive and selective diagnosis of infectious diseases, the sample preparation step should be eliminated with further improvement to obtain a fully POC sample-to-result platform.

In another study, a label-free electrochemical biosensor was proposed for COVID-19-specific viral RNA/c-DNA [141]. The biosensor consisted of a three-electrode system including platinum electrodes as a reference and a counter electrode and a titanium substrate which was functionalized with AuNPs via electrodeposition as a working electrode. At first, a complementary single-stranded probe with a thiol end was constructed. Then, the modified probe was immobilized to the gold surface via gold-thiol interaction. The hybridization occurred after the target nucleotide was attached to the 
biosensor surface. While the reaction chamber was confined with a PDMS reservoir, a USB was integrated into the handheld biosensor to allow specific DNA detection of COVID19. Smartphones with software applications were used to analyze electrochemical data. The drawback of this method includes the need for the target DNA/RNA to be extracted from patients for sample preparation. The biosensor is under development and differential pulse voltammetry and electrochemical impedance spectroscopy techniques can be applied to attain an electrochemical response of DNA sensors [142, 143].

In addition to the aforementioned biosensors, localized surface plasmon resonance (LSPR) biosensors have also been used for COVID-19. LSPR detection systems exhibit higher sensitivity compared to other biosensors due to the improved plasmonic field [144]. Hence, LSPR has the potential for label-free and real-time biosensing of nano/micro scale analytes. An optical biosensor was recently developed based on real-time dual-functional plasmonic photothermal (PPT) for the detection of SARS-CoV [145]. Nucleic acid hybridization of SARS-CoV-2 RNA sequences was performed using gold nanoisland (AuNI) chips functionalized with a DNA probe. At first, Au nanofilm was used to construct the AuNI sensor chips on the glass surface. Then, the solution containing thiol-cDNA of RdRp-COVID was gradually injected into the sensor chip to functionalize the chip, forming Au-S bond between the thiol-cDNA and AuNIs. The optimum cDNA concentration was found as $1 \mathrm{nmol}$ for modification of the AuNI chips. After that, the AuNI chips were placed into LSPR systems to detect specific viral sequences. To observe PPT heat effect, the thermoplasmonic heat was in situ generated on the surface of AuNIs chip under the PPT heat $(32 \mathrm{~mW}$ optical power at $532 \mathrm{~nm}$ ) and hybridization reaction was occurred. Once the RdRp-COVID RNAs were introduced to the microfluidic chip, the LSPR response of the dual-functional AuNI biosensor increased. It was observed that nucleic acid hybridization kinetics of RdRp-COVID and its cDNA was improved by the localized PPT effect. Thus, the sensitivity of the biosensor was improved due to the combination of the LSPR sensing transduction and the plasmonic photothermal (PPT) influence. With this biosensor, the detection limit of the $\mathrm{RdRp}$ gene was about $0.22 \mathrm{pM}$. In addition, $\mathrm{RdRp}$ genes were successfully distinguished from SARS-CoV and SARS-CoV2 , showing the high specificity of the biosensor.

\section{Paper-based POC biosensors for the detection of nucleic acids}

Besides chip-based biosensors, paper-based biosensors have drawn attention for diagnosis of COVID-19 due to their costeffectiveness, simple fabrication, and operation which provide rapid diagnosis at home. Lateral flow test strips, in particular, have been used for nucleic acid detection as the immobilized probes provide a visual readout upon interaction with target nucleic acids [146, 147].
Numerous studies have reported the use of lateral flow test strips to detect nucleic acid of COVID-19 based on colorimetric detection. For instance, one study introduced a multiplex reverse transcription LAMP (mRT-LAMP) coupled with a lateral flow test strip [148] (Fig. 6a). Briefly, in a single tube, the opening reading frame $1 \mathrm{a} / \mathrm{b}(\mathrm{ORF} 1 \mathrm{ab})$ and nucleoprotein (N) target genes of SARS-CoV-2 were amplified utilizing two LAMP primer sets. Fluorescein (FITC)-/digoxin- and biotinattached duplex amplicons were generated due to the presence of FITC-/digoxin- and biotin-labeled primers on the nitrocellulose membrane. Then, the ORF1ab and N genes of SARS$\mathrm{CoV}-2$ were simultaneously detected by the appearance of a red band on the test strip as a result of interaction between a biotin-labeled product and streptavidin-conjugated colored nanoparticles in $2 \mathrm{~min}$. The detection limit was 12 copies each of the ORF1ab-plasmid and N-plasmid constructs, which were compatible with results obtained by singleplex detection. The assay was validated using clinical samples from 33 patients infected by SARS-CoV-2 and 96 non-SARS-CoV-2 infected patients. However, the number of clinical samples was limited and sputum, blood, urine, feces, and nasal samples were not utilized to determine COVID-19 infection.

Besides colorimetric detection, paper-based biosensors have also been introduced to detect nucleic acids of COVID19 based on fluorescence detection. For example, a CRISPRCas 12 technology [140, 150]. Generally, Clustered Regularly Interspaced Short Palindromic Repeats (CRISPR/Casbased) have been developed for nucleic acid detection. The singlestranded DNA (ssDNA) reporters are cleaved by Cas 12 once the target is introduced, producing fluorescent signals [151, 152]. For example, Lucia et al. [153] developed a portable and sensitive detection method for COVID-19 using CRISPRCas12a. At first, the SARS-CoV-2 fragments corresponding to the RdRp, ORF1b and ORF1ab genes were synthesized based on the WH-human1 sequence (GenBank MN908947). Then, the TwistAmp® Basic recombinase polymerase amplification (RPA) kit was used to employ the one-step target amplification for $30 \mathrm{~min}$ at $42^{\circ} \mathrm{C}$, followed by the generation of the CRISPR-detection complex reaction for $10 \mathrm{~min}$ at room temperature. Commercially available Milenia HybriDetect (TwistDx) lateral flow test strip was used for detection of the complex. A saliva sample from a healthy donor spiked with synthetic SARS-CoV-2 RNA fragments was also tested. According to results produced by the fluorescence spectrophotometer, the limit of detection for ORF1ab coronavirus sequences was about 10 copies $/ \mu \mathrm{L}$. Yoshimi et al. [154] developed a Cas3-based (SHERLOCK, Specific High-sensitive Enzymatic Reporter UnLOCKing) lateral flow assay for POC detection of SARS-CoV-2. Cas3-Operated Nucleic Acid detectioN (CONAN) as a nucleic acid detection method along with isothermal amplification methods offers a fast, sensitive detection platform for SARS-CoV-2 without the necessity of an instrument. In the presence of targets, the cleaved 
a

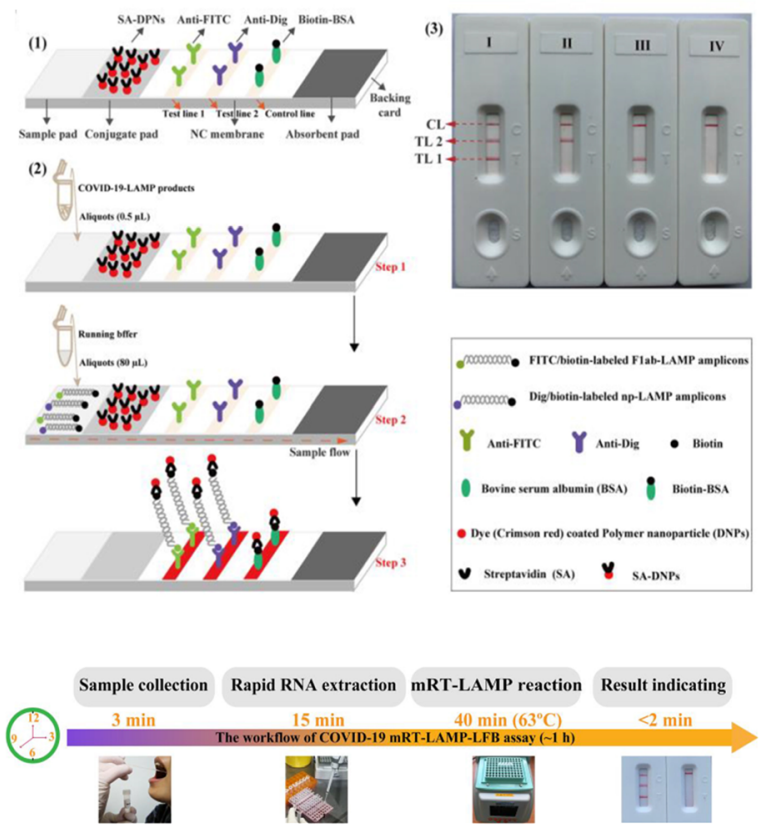

b

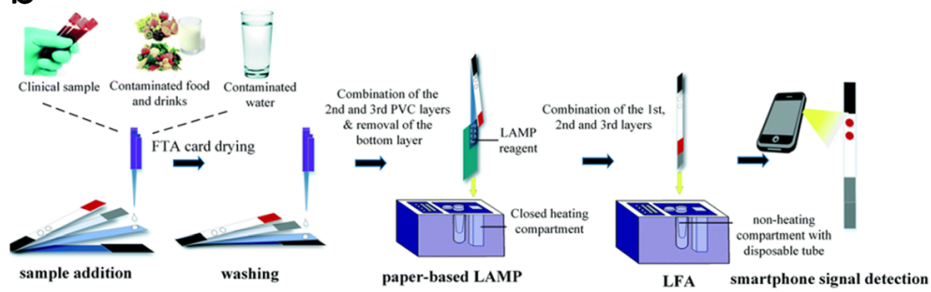

C 1

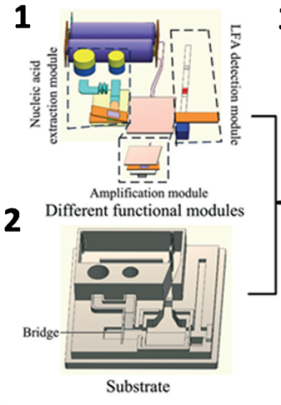

3

3 Buson

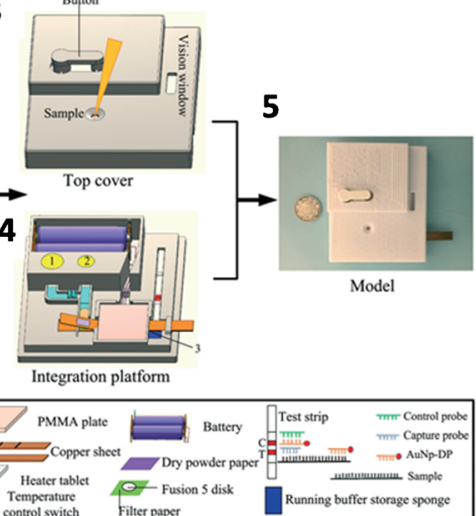

Fig. 6 Paper-based biosensors for potential nucleic acid detection for COVID-19. a A paper-based biosensor or lateral flow test strip for visualization of COVID-19 reverse transcription loop-mediated isothermal amplification (RT-LAMP) products. Adapted with permission from

fluorophore-quencher (FQ)-labeled ssDNA probe $\left(5^{\prime}-15-\right.$ FITC/TAGCATGTCA/3-Biotin/-3') leads to a signal for positive results on lateral flow strip. A detection limit of $<10^{2}$ copies for SARS-CoV-2 was obtained using CONANLAMP at $62^{\circ} \mathrm{C}$ for $30 \mathrm{~min}$. The paper-based biosensor was validated using COVID-19 patients from nasopharyngeal swabs and the fluorescence signal was detected in a few min. Another group developed a simple SHERLOCK Testing in One Pot (STOP) that performs amplification at a single temperature to detect SARS-CoV-2 [155]. Briefly, a nasopharyngeal swab or saliva sample was added into the lysis buffer and heated at $60^{\circ} \mathrm{C}$. Then, a commercial lateral flow test strip was used to detect synthetic SARS-CoV-2 genomes in a reaction tube for 2 min lateral flow readout. A detection limit of 100 copies of the viral genome was obtained. While the aforementioned target detection utilizes inexpensive and simple lateral flow test strips, the step of nucleic acid extraction is off-chip, which is complex and equipmentdependent; hence, these biosensors are less suitable for POC applications. Therefore, there is a need to develop an integrated paper-based biosensor for rapid nucleic acid testing for COVID-19.

Several studies have demonstrated the development of sample-to-answer paper-based biosensors which offer tremendous potential for diagnosis of COVID-19. For instance, Rodriguez et al. [156] reported a foldable paper-based biosensor combining nucleic acid extraction, LAMP, and lateral flow

detection using immunochromatographic strips for POC detection of nucleic acids. Briefly, a polyethersulfone (PES) membrane was used to perform LAMP assay. FITC and biotin were utilized to label forward and reverse loop primers in order to detect amplified products on test strips that include streptavidin-conjugated AuNPs. The signal was then captured and analyzed using an iPhone 5 camera and ImageJ, respectively. The biosensor provides rapid and POC detection of human papillomavirus DNAs from the patient cervical samples in no more than an hour. This platform also can be adapted for the diagnosis of any diseases by applying corresponding primer probes and LAMP assay conditions. However, the disadvantage of the biosensor is that an external heat source is necessary for the isothermal amplification step. In addition, assay conditions should be further optimized to decrease the volume needed and pipetting steps of solutions. Thus, the portability and ease of use by the end user should be improved. This study was improved by Choi et al. [149], developing a paper-based biosensor integrating nucleic acid extraction, amplification, and colorimetric detection using a smartphone for the detection of nucleic acid (Fig. 6b). The biosensor includes four layers where fluid flow is controlled by polyvinyl chloride (PVC) layers. After the sample addition and nucleic acid extraction were performed on a Fast Technology Analysis (FTA) card, amplification reagents were loaded onto the glass fiber for amplification. A portable and battery-powered heating biosensor was used for the LAMP 
step. Finally, the double-stranded DNAs were separated into single strands at $95^{\circ} \mathrm{C}$ followed by hybridization between the thiolated probe and synthesized AuNPs. A smartphone was used to capture colorimetric images and the color intensities were analyzed by a custom App developed by the group. The biosensor was applied to detect Escherichia coli in drinking water, milk, blood, and spinach samples with a detection limit of $10-1000 \mathrm{CFU} \mathrm{mL} \mathrm{m}^{-1}$. The analysis time from the sample-toanswer process takes $\sim 1 \mathrm{~h}$. The advantage of this biosensor is nucleic acid extraction, amplification, and testing processes that require various temperature and duration for each layer can be performed with the use of a single biosensor containing a heater, PVC layers as valves, and a suitable compartment to insert a microcentrifuge tube for lateral flow assay (LFA). Also, the proposed biosensor is suitable for POC diagnosis due to the ease of signal readout by the naked eye. This biosensor was improved by eliminating the necessity of off-chip nucleic acid extraction, amplification, and reagent storage as well as benchtop equipment. With further improvement of the previous biosensor, Tang et al. [25] presented a fully disposable paper-based biosensor that combines nucleic acid extraction, helicase-dependent isothermal amplification (tHDA), and lateral flow assay for nucleic acid testing (Fig. 6c). Once the sample was placed in the sample zone, the extraction step was triggered by releasing lysis buffer via pushing the button on the biosensor. Then, the two copper sheets including the DNA template and freeze-dried thermophilic tHDA reagents were simultaneously placed in the amplification zone to start the amplification step. The isothermal amplification was achieved with the use of an integrated battery, an ultrathin heater, a temperature control switch along with a dried enzyme mixture which is stored on-chip. The target and running buffer diffused through the lateral flow strip by capillary action after the copper sheets were placed in the LFA detection compartment. Finally, a colorimetric signal was observed with the naked eye using the lateral flow test strip. An iPhone 6S was used to capture the images of the test line and the ImagePro Plus 6.0 was used for the analysis of the optical densities of the test line. The developed biosensor is advantageous over the integrated LFA biosensor $[156,157]$ based on PES-based DNA/RNA extraction, real-time amplification, and detection process as it does not require an external heater for the amplification step. It is promising for POC detection of COVID-19 while meeting the ASSURED criteria.

\section{Other POC biosensors for the detection of nucleic acids}

In addition to the chip-based and paper-based POC biosensors, other biosensors such as thread-based biosensors and emerging nanomaterial-based biosensors have also been used for COVID-19. For example, a nanomaterial and circle-tocircle amplification (C2CA)-based optomagnetic biosensor for real-time detection of SARS-CoV-2 was developed by
Tian et al. [158] (Fig. 7a). The method provides a homogeneous and isothermal nucleic acid quantification using $\mathrm{C} 2 \mathrm{CA}$ and optomagnetic analysis of magnetic nanoparticle (MNP) strategy. This biosensor is advantageous over previously reported C2CA-based sensors as it offers real-time and simple operation due to the combination of two amplification rounds in a single-step process. The biosensor exhibited a detection limit of $0.4 \mathrm{fM}$ for the detection of a synthetic SARS-CoV-2 RdRp DNA with a total assay time of $\sim 100 \mathrm{~min}$.

In another study, a magnetic bead-quantum dot (MBQdot)-CRISPR DNA detection biosensor was developed by Bao et al. [161]. After the biotinylated ssDNA probe was cleaved by Cas enzyme, the probe molecules were attached to streptavidin-coated magnetic particles. Then, the quantum dot was used as a fluorophore-quencher substrate to conjugate complementary ssDNA oligonucleotide molecules. Once uncleaved probes linked to the magnetic beads were hybridized by the quencher molecules, the cleaved ssDNA probes attached to the Qdots were separated via magnetic separation. The resulting signal was detected fluorometrically for a target double-stranded DNA of the African Swine Fever Virus (ASFV) genome and a detection limit of $0.5 \mathrm{nM}$ was obtained under a UV flashlight. Compared to traditional CRISPR-Cas technology, the proposed method showed low detection background due to the isolation of magnetic beads that lead to eliminating background signal. Multiplex detection of DNA and RNA targets can be achieved by this method. Although the developed method offers a simple, instrument-free, onepot reaction for amplification reagents which is suitable for resource-limited countries at POC detection of viruses, it has not been tested for COVID-19 yet. Another nanomaterialbased biosensor for POC detection was presented by Gong et al. [159] (Fig. 7b). Green colored-core upconverting nanoparticles (UCNPs) (NaYF4:Yb/Er) were synthesized and UCNP-DNA probe was used to modify the nitrocellulose membrane. The detection sensitivity of biosensors with UCNPs was higher than that of AuNPs. The working principle of this biosensor is based on the reaction between UCNP probes and target nucleic acid followed by recognition of capture probes at the test line while capturing the excess free UCNP probes at the control line. Once the specimen was introduced to the biosensor for detection of nucleic acids of hepatitis B virus, it was observed that fluorescent signal at the test line increased with increasing the number of targets in the sample. Fluorescence intensities were quantitatively analyzed with the use of UCNP-LFA reader and the smartphone with a custom-designed App in a real-time manner. The proposed biosensor meets the requirement of clinical diagnosis as it presents 5-10-fold lower detection limit compared with the clinical cutoff value. This biosensor is advantageous over some existing biosensors as the universality of the platform was demonstrated by quantitative detection of various analytes such as ochratoxin $\mathrm{A}, \mathrm{Hg}^{2+}$, salmonella, hepatitis B 
a

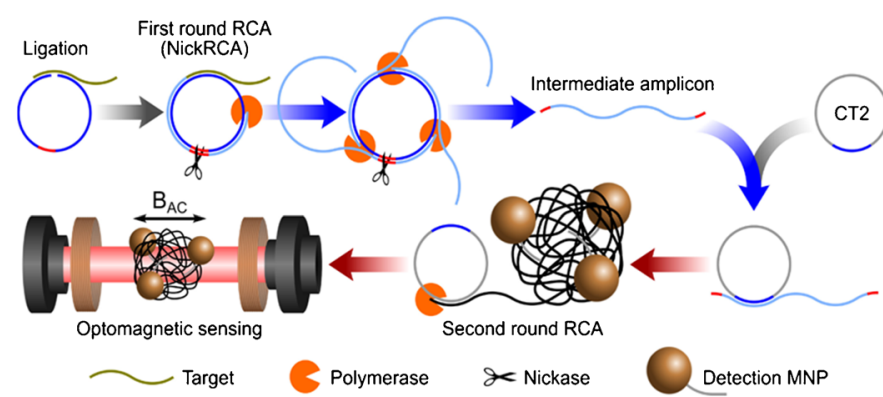

C

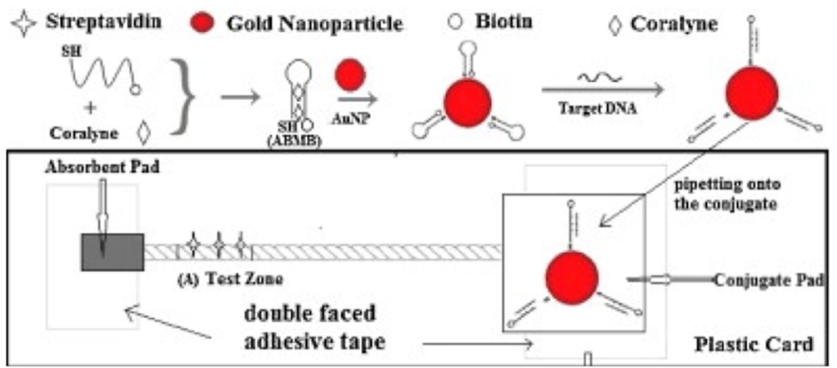

Fig. 7 Other point-of-care biosensors for potential nucleic acid detection for COVID-19. a An optomagnetic biosensor for real-time nucleic acid detection of SARS-CoV-2. Adapted with permission from [158]. b Schematic diagram of an upconverting nanoparticle-based biosensor for

virus, and growth stimulation expressed gene 2, ST-2. Also, the biosensor is promising for POC detection of COVID-19 due to its miniaturization and portability.

Furthermore, a portable colorimetric LAMP (qcLAMP)based biosensor was constructed for quantitative detection of SARS-CoV-2 [162]. The miniaturized biosensor was fabricated using 3D printing technology that allows visual readout of the color change during DNA amplification via a smartphone application. The biosensor exhibited a low detection limit (110 copies of DNA) within less than $30 \mathrm{~min}$. The validation of the method was demonstrated with the use of clinically confirmed positive and negative saliva and tissue samples. The biosensor is rapid and cost-effective which is highly suitable for POC COVID-19 diagnosis.

In addition, thread-based biosensors have also been introduced for nucleic acid detection which are more advantageous than the traditional 96-well plates due to lower sample/ reagents consumption and shorter analysis time [163]. For instance, a cotton thread biosensor based on fluorescence detection was developed by Du et al. [160] (Fig. 7c). While a single cotton thread strip was placed on two parallel doublefaced adhesive tapes, an absorbent pad was positioned at the downstream point. The detection mechanism was based on the interaction between AuNP conjugates modified with biotinylated adenosine-based molecular beacon (ABMB) probes and the sample containing target DNA sequences. In the presence b
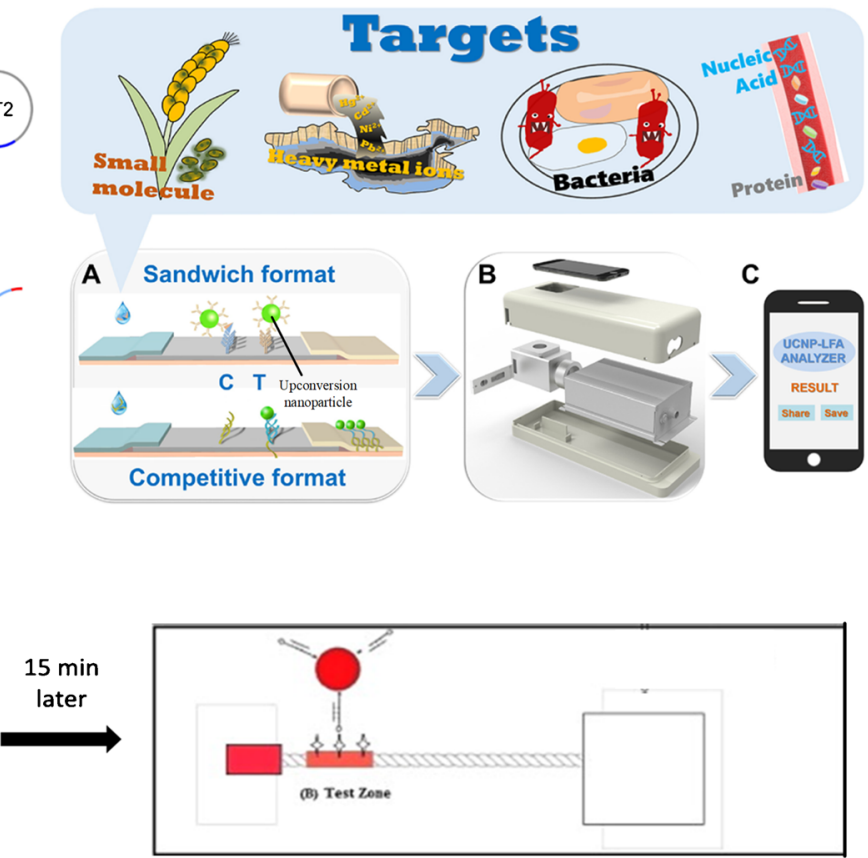

pathogen detection. Adapted with permission from [159]. c A cotton thread-based biosensor for nucleic acid detection for disease diagnosis. Adapted with permission from [160]

of targets, the biotin group of the probes reacted with streptavidin attached to the cotton thread resulting in a red band. The color intensities on the test zone were quantitatively analyzed using a scanner and ImageJ software. The threadbased biosensor could detect human genetic disease, hereditary tyrosinemia type I-related DNA in a linear range of 2.5$100 \mathrm{nM}$. This biosensor shows great promise for the detection of COVID-19 owing to its simple fabrication steps and costeffectiveness.

\section{Commercial POC biosensors for COVID-19}

To date, several cost-effective POC biosensors have been commercialized for COVID-19, which mainly detects nucleic acids and antibodies (Table 2). For instance, HybriDetect, a paper-based biosensor or lateral flow test strip, has been developed by Milenia Biotec GmbH for the detection of RPA, LAMP, and PCR amplicons. Following amplification with specific primers, the FITC-tagged amplicons can be detected using HybriDetect dipstick based on AuNPs. The result can then be observed within 5 min with the naked eye $[140,155]$. Another paper-based biosensor developed by Abingdon Health's PCRD is also based on nucleic acid lateral flow immunoassay. It is developed in the form of cassettes and dipsticks (PCRD FLEX) that can give results within $10 \mathrm{~min}$ 


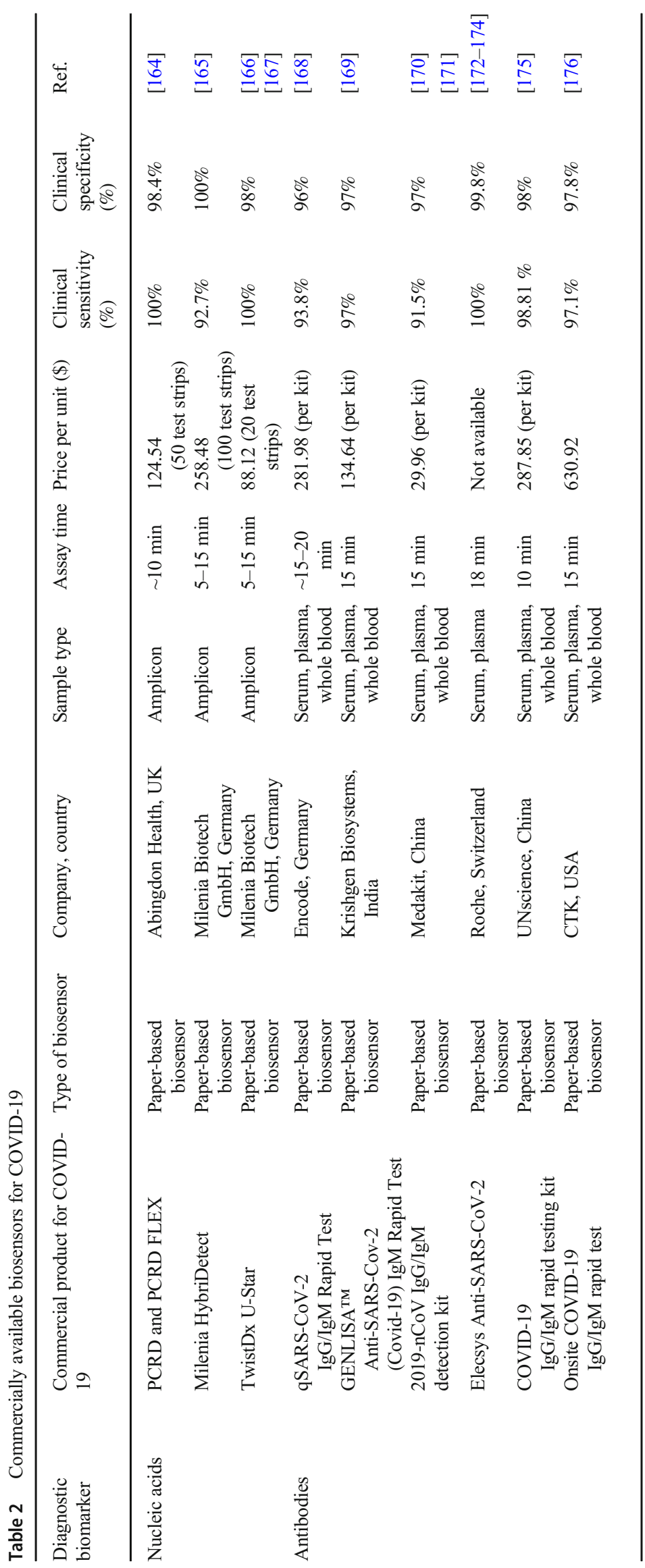


which can potentially be used for COVID-19. On the other hand, a U-Star disposable nucleic acid-based lateral flow detection unit has been developed by TwistDx. Briefly, this test strip is designed to detect a biotin and FITC or FAM-labeled amplicon. The process of detection occurs in a sealed cartridge, which gives results within 10-15 min [177, 178].

Besides nucleic acid detection, some commercial biosensors have been used for the detection of antibodies (IgG/IgM). Lateral flow test strips, in particular, have been widely available in the market to rapidly diagnose COVID-19 based on $\mathrm{IgG} / \mathrm{IgM}$. For instance, Cellex's qSARS-CoV-2 IgG/IgM Rapid Test was developed by Cellex, which is able to produce results within 20 min using different samples from COVID-19 patients like the serum, plasma, or whole blood samples. The test strips consist of (i) a conjugate pad containing SARS$\mathrm{CoV} 2$ recombinant antigen conjugated with AuNPs and rabbit IgG gold conjugate, and (ii) a nitrocellulose membrane having an IgM and IgG lines coated with anti-human IgM and antihuman IgG, respectively. There is also a control line coated with goat anti-rabbit IgG. The clinical sensitivity and specificity of the kit are 93\% and 96\%, respectively [168]. Abbexa's COVID-19 IgG/IgM rapid testing kit was used to detect IgG/ IgM, producing test results in $10 \mathrm{~min}$. Similar to Cellex's test kit, the samples such as the whole blood, serum, and plasma can be used for testing, which produces colorimetric IgG and IgM signals in two separate bands. The clinical sensitivity and specificity of the kit are $93 \%$ and $96 \%$, respectively [175, 179]. In addition, Onsite COVID-19 IgG/IgM rapid test has been developed by CTK, USA, for screening anti-SARS$\mathrm{CoV} 2 \mathrm{IgG}$ and IgM from different samples like the serum, plasma, or whole blood in $15 \mathrm{~min}$. The clinical sensitivity and specificity of the kit are $97.1 \%$ and $97.8 \%$, respectively $[176,180]$. These kits have enabled the rapid diagnosis of patients with suspected COVID-19 infection to manage the ongoing pandemic.

\section{Conclusion and future perspectives}

In summary, given that there are no specific vaccines or treatments for COVID-19, rapid and early diagnosis is imperative in providing timely health measures to reduce the risk of health complications and to prevent further spread of the virus. This review article presents an overview of the development of POC biosensors, including chip-based biosensors, paper-based biosensors, and other biosensors (e.g., textile-based and nanomaterialbased biosensors) for COVID-19. These biosensors are simple, user-friendly, and cost-effective, holding a significant potential to substitute the conventional lengthy and time-consuming technologies. Antibody tests are appropriate to detect late-stage or past infections while nucleic acid tests are effective in identifying the early stage of infection. While the current nucleic acid tests present a higher sensitivity and specificity than that of antibody tests, they involve more lengthy and complicated processes (i.e., nucleic acid extraction, amplification, and detection). In contrast, even though the existing antibody tests show lower specificity, they have shorter turnaround time than that of nucleic acid tests, which enable fast decision-making and health management in remote settings.

While POC biosensors are widely available, further improvements are required to bring the technology to the next level. The recent commercial diagnostic kits that involve the use of $\mathrm{IgG} / \mathrm{IgM}$ test strips have helped shorten the assay time but their sensitivity and specificity are yet to be improved [181]. To enhance detection sensitivity, some sensitivity enhancement strategies should be implemented into the biosensors such as fluidic control strategies [182], enzyme-based signal improvement strategies [183], and sample concentration strategies [184]. In addition to detection sensitivity, the capability of multiplexing is essential to increase assay productivity [185]. In particular, the combination of both $\mathrm{IgG} /$ $\mathrm{IgM}$ and nucleic acid tests would allow the detection of both early and late stages of COVID-19 infection, yielding more accurate and reliable results.

Apart from that, to simplify user steps, multiple processing steps ranging from sample preparation to signal detection should be integrated into a single biosensor. Specifically, recent studies have incorporated sample-to-answer processes into a single biosensor to detect antibodies and nucleic acids of pathogens, which could be applied for COVID-19 [186]. Furthermore, incorporating a sample collector into the biosensors would make it more user-friendly. For instance, incorporating a microneedle for the painless collection of blood samples would make the biosensor simpler and would also reduce patient anxiety and stress [187]. Given that blood samples can be collected with a single push of a button, the use of this selfcontained biosensor only requires minimal training. In addition to blood testing, more POC biosensors should be developed to screen the COVID-19 virus in saliva [188]. This approach is non-invasive and the sample is easy to collect, transport, and analyze.

Future work should also focus on developing portable and equipment-free biosensors. Preserving reagents on-chip would be preferable to eliminate the need for sophisticated storage units (e.g., fridge and freezer) [189]. Furthermore, incorporating portable power sources (e.g., batteries) would advance assay functionality, especially for nucleic acid tests that require a powered heater for amplification $[25,190]$. This function is useful particularly in settings where high-end equipment and electricity are unavailable. Data quantification is also critical to accurately evaluate patients' health conditions [28]. It could be achieved through developing smartphone apps that allow data analysis in remote settings while enabling data storage to track patients' health status. The use of Internet-of-Things allows patients to share test results with healthcare workers, and facilitates analysis and 
tracking of patient health information for onsite real-time health monitoring [191, 192].

In short, the simplicity, rapidity, cost-effectiveness, and portability of the biosensors play a key role in POC testing as the pandemic evolves. Given that the virus can be transmitted by an asymptomatic person, the development of homebased POC biosensors is of paramount importance to allow self-testing and immediate self-isolation once tested positive to avoid further transmission of the virus. It is envisioned that the emerging POC biosensors could be produced on a large scale for mass testing to rapidly identify COVID-19 cases and manage the pandemic.

\section{Declarations}

Competing interests The authors declare no competing interests.

\section{References}

1. Ting DSW, Carin L, Dzau V, Wong TY. Digital technology and COVID-19. Nat Med. 2020;26:459-61.

2. Wang Z, Tang K. Combating COVID-19: health equity matters. Nat Med. 2020;26:458-458.

3. He X, Lau EH, Wu P, Deng X, Wang J, Hao X, et al. Temporal dynamics in viral shedding and transmissibility of COVID-19. Nat Med. 2020;26:672-5.

4. Yang P, Wang X. COVID-19: a new challenge for human beings. Cell Mol Immunol. 2020;17:555-7.

5. Menni C, Valdes AM, Freidin MB, Sudre CH, Nguyen LH, Drew $\mathrm{DA}$, et al. Real-time tracking of self-reported symptoms to predict potential COVID-19. Nat Med. 2020:1-4.

6. Lipsitch M, Kahn R, Mina MJ. Antibody testing will enhance the power and accuracy of COVID-19-prevention trials. Nat Med. 2020:1-2.

7. GeurtsvanKessel CH, Okba NM, Igloi Z, Bogers S, Embregts CW, Laksono BM, et al. An evaluation of COVID-19 serological assays informs future diagnostics and exposure assessment. Nat Commun. 2020;11:1-5.

8. Mei X, Lee H-C, Diao K, Huang M, Lin B, Liu C, et al. Artificial intelligence-enabled rapid diagnosis of patients with COVID-19. Nat Med. 2020:1-5.

9. Tang Y-W, Schmitz JE, Persing DH, Stratton CW. Laboratory diagnosis of COVID-19: current issues and challenges. J Clin Microbiol. 2020;58:6.

10. Ai T, Yang Z, Hou H, Zhan C, Chen C, Lv W, et al. Correlation of chest CT and RT-PCR testing for coronavirus disease 2019 (COVID-19) in China: a report of 1014 cases. Radiology. 2020 Aug;296(2):E32-40.

11. Won J, Lee S, Park M, Kim TY, Park MG, Choi BY, et al. development of a laboratory-safe and low-cost detection protocol for SARS-CoV-2 of the coronavirus disease 2019 (COVID-19). Exp Neurobiol. 2020 Apr 30;29(2):107-19.

12. Yu L, Wu S, Hao X, Li X, Liu X, Ye S, et al. Rapid colorimetric detection of COVID-19 coronavirus using a reverse transcriptional loop-mediated isothermal amplification (RT-LAMP) diagnostic plat-form: iLACO [Internet]. Infectious Diseases (except HIV/AIDS); $2020 \mathrm{Feb}$ [cited $2020 \mathrm{Jul}$ 27]. Available from: http://medrxiv.org/lookup/doi/10.1101/2020.02.20.20025874.

13. Xia N-S, Wang G-Q, Gong W-F. Serological test is an efficient supplement for detecting RNA to confirm SARS-CoV-2 infection
[Internet]. Medicine \& Pharmacology; 2020 Apr [cited 2020 Aug 22]. Available from: https://www.preprints.org/manuscript/ 202003.0184/v2.

14. Xiang J, Yan M, Li H, Liu T, Lin C, Huang S, et al. Evaluation of enzyme-linked immunoassay and colloidal goldimmunochromatographic assay kit for detection of novel coronavirus (SARS-Cov-2) causing an outbreak of pneumonia (COVID19) [Internet]. Epidemiology; 2020 Mar [cited 2020 Jul 27]. Available from: http://medrxiv.org/lookup/doi/10.1101/2020.02. 27.20028787.

15. Li Z, Yi Y, Luo X, Xiong N, Liu Y, Li S, et al. Development and clinical application of a rapid IgM-IgG combined antibody test for SARS-CoV-2 infection diagnosis. J Med Virol. 2020 Feb 27: jmv.25727.

16. Pan Y, Li X, Yang G, Fan J, Tang Y, Zhao J, et al. Serological immunochromatographic approach in diagnosis with SARS-CoV2 infected COVID-19 patients. Journal of Infection. 2020 Jul;81(1):e28-32.

17. Howson ELA, Kurosaki Y, Yasuda J, Takahashi M, Goto H, Gray AR, et al. Defining the relative performance of isothermal assays that can be used for rapid and sensitive detection of foot-andmouth disease virus. J Virol Methods [Internet]. 2017 Nov 1 [cited 2020 Sep 20];249:102-110. Available from: http://www. sciencedirect.com/science/article/pii/S016609341730335X.

18. Yang W, Yan F. Patients with RT-PCR-confirmed COVID-19 and normal chest CT. Radiology. 2020;295:E3-E3.

19. Bastos ML, Tavaziva G, Abidi SK, Campbell JR, Haraoui L-P, Johnston JC, et al. Diagnostic accuracy of serological tests for covid-19: systematic review and meta-analysis. bmj. 2020;370.

20. Bernheim A, Mei X, Huang M, Yang Y, Fayad ZA, Zhang N, et al. Chest CT findings in coronavirus disease-19 (COVID-19): relationship to duration of infection. Radiology. 2020;200463.

21. Choi JR, Hu J, Wang S, Yang H, Wan Abas WAB, PingguanMurphy B, et al. based point-of-care testing for diagnosis of dengue infections. Crit Rev Biotechnol. 2017;37:100-11.

22. Park J, Han DH, Park J-K. Towards practical sample preparation in point-of-care testing: user-friendly microfluidic devices. Lab Chip. 2020;20:1191-203.

23. Yew CHT, Azari P, Choi JR, Muhamad F, Pingguan-Murphy B. Electrospun polycaprolactone nanofibers as a reaction membrane for lateral flow assay. Polymers. 2018;10:1387.

24. Choi JR. Development of point-of-care biosensors for COVID-19. Front Chem. 2020;8:517.

25. Tang R, Yang H, Gong Y, You M, Liu Z, Choi JR, et al. A fully disposable and integrated paper-based device for nucleic acid extraction, amplification and detection. Lab Chip. 2017;17:1270-9. https://doi.org/10.1039/C6LC01586G.

26. Chinnadayyala SR, Park J, Le HTN, Santhosh M, Kadam AN, Cho S. Recent advances in microfluidic paper-based electrochemiluminescence analytical devices for point-of-care testing applications. Biosens Bioelectron. 2019;126:68-81.

27. Hu J, Choi JR, Wang S, Gong Y, Feng S, Pingguan-Murphy B, et al. Multiple test zones for improved detection performance in lateral flow assays. Sens Actuators B Chem. 2017;243:484-8.

28. Liu J, Geng Z, Fan Z, Liu J, Chen H. Point-of-care testing based on smartphone: the current state-of-the-art (2017-2018). Biosens Bioelectron. 2019;132:17-37.

29. Yew C-HT, Azari P, Choi JR, Li F, Pingguan-Murphy B. Electrospin-coating of nitrocellulose membrane enhances sensitivity in nucleic acid-based lateral flow assay. Anal Chim Acta. 2018;1009:81-8.

30. Gowri A, Ashwin Kumar N, Suresh Anand BS. Recent advances in nanomaterials based biosensors for point of care (PoC) diagnosis of COVID-19 - a minireview. TrAC Trends Anal Chem. 2021;137:116205. https://doi.org/10.1016/j.trac.2021.116205. 
31. Weiss C, Carriere M, Fusco L, Capua I, Regla-Nava JA, Pasquali $\mathrm{M}$, et al. Toward nanotechnology-enabled approaches against the COVID-19 pandemic. ACS Nano. 2020;14:6383-406. https://doi. org/10.1021/acsnano.0c03697.

32. Srivastava M, Srivastava N, Mishra PK, Malhotra BD. Prospects of nanomaterials-enabled biosensors for COVID-19 detection. Sci Total Environ. 2021;754:142363. https://doi.org/10.1016/j. scitotenv.2020.142363.

33. Iravani S. Nano- and biosensors for the detection of SARS-CoV2: challenges and opportunities. Mater Adv. 2020;1:3092-103. https://doi.org/10.1039/D0MA00702A.

34. Leïchlé T, Nicu L, Alava T. MEMS biosensors and COVID-19: missed opportunity. ACS Sens. 2020;5:3297-305. https://doi.org/ 10.1021/acssensors.0c01463.

35. Carter LJ, Garner LV, Smoot JW, Li Y, Zhou Q, Saveson CJ, et al. Assay techniques and test development for COVID-19 diagnosis. ACS Cent Sci. 2020;6:591-605. https://doi.org/10.1021/ acscentsci.0c00501.

36. Pokhrel $\mathrm{P}, \mathrm{Hu} \mathrm{C}, \mathrm{Mao} \mathrm{H}$. Detecting the Coronavirus (COVID-19). ACS Sens. 2020;5:2283-96. https://doi.org/10.1021/acssensors. 0c01153.

37. Udugama B, Kadhiresan P, Kozlowski HN, Malekjahani A, Osborne M, Li VY, et al. Diagnosing COVID-19: the disease and tools for detection. ACS Nano. 2020;14:3822-35.

38. Cui F, Zhou HS. Diagnostic methods and potential portable biosensors for coronavirus disease 2019. Biosens Bioelectron. 2020;165:112349. https://doi.org/10.1016/j.bios.2020.112349.

39. Morales-Narváez E. The impact of biosensing in a pandemic outbreak: COVID-19. Biosens Bioelectron. 2020;6.

40. Santiago I. Trends and innovations in biosensors for COVID-19 mass testing. Chembiochem. 2020;21:2880-9. https://doi.org/10. 1002/cbic.202000250.

41. Tymm C, Zhou J, Tadimety A, Burklund A, Zhang JXJ. Scalable COVID-19 detection enabled by lab-on-chip biosensors. Cell Mol Bioeng. 2020;13:313-29. https://doi.org/10.1007/s12195-02000642-z.

42. Xu L, Li D, Ramadan S, Li Y, Klein N. Facile biosensors for rapid detection of COVID-19. Biosens Bioelectron. 2020;170:112673. https://doi.org/10.1016/j.bios.2020.112673.

43. Bhalla N, Pan Y, Yang Z, Payam AF. Opportunities and challenges for biosensors and nanoscale analytical tools for pandemics: COVID-19. ACS Nano. 2020;14:7783-807. https://doi. org/10.1021/acsnano.0c04421.

44. Chen L, Zhang G, Liu L, Li Z. Emerging biosensing technologies for improved diagnostics of COVID-19 and future pandemics. Talanta. 2021;225:121986. https://doi.org/10.1016/j.talanta.2020. 121986.

45. Parihar A, Ranjan P, Sanghi SK, Srivastava AK, Khan R. Pointof-care biosensor-based diagnosis of COVID-19 holds promise to combat current and future pandemics. ACS Appl Bio Mater. 2020;3:7326-43. https://doi.org/10.1021/acsabm.0c01083.

46. Hussein HA, Hassan RYA, Chino M, Febbraio F. Point-of-care diagnostics of COVID-19: from current work to future perspectives. Sensors. 2020;20:4289. https://doi.org/10.3390/s20154289.

47. Rezaei M, Razavi Bazaz S, Zhand S, Sayyadi N, Jin D, Stewart MP, et al. Point of care diagnostics in the age of COVID-19. Diagnostics. 2021;11:9. https://doi.org/10.3390/ diagnostics11010009.

48. Suleman S, Shukla SK, Malhotra N, Bukkitgar SD, Shetti NP, Pilloton R, et al. Point of care detection of COVID-19: advancement in biosensing and diagnostic methods. Chem Eng J. 2021;414:128759.

49. Furukawa NW, Brooks JT, Sobel J. Evidence supporting transmission of severe acute respiratory syndrome coronavirus 2 while presymptomatic or asymptomatic. Emerg Infect Dis. 2020;26. https://doi.org/10.3201/eid2607.201595.
50. Guo Z-D, Wang Z-Y, Zhang S-F, Li X, Li L, Li C, et al. Aerosol and surface distribution of severe acute respiratory syndrome coronavirus 2 in hospital wards, Wuhan, China, 2020. Emerg Infect Dis. 2020;26:1583-91. https://doi.org/10.3201/eid2607.200885.

51. Li Q, Guan X, Wu P, Wang X, Zhou L, Tong Y, et al. Early transmission dynamics in Wuhan, China, of novel coronavirusinfected pneumonia. N Engl J Med. 2020;382:1199-207. https:// doi.org/10.1056/NEJMoa2001316.

52. McGrath BA, Brenner MJ, Warrillow SJ, Pandian V, Arora A, Cameron TS, et al. Tracheostomy in the COVID-19 era: global and multidisciplinary guidance. Lancet Respir Med. 2020.

53. Wang Q, Zhang Y, Wu L, Niu S, Song C, Zhang Z, et al. Structural and functional basis of SARS-CoV-2 entry by using human ACE2. Cell. 2020;181:894-904.e9. https://doi.org/10. 1016/j.cell.2020.03.045.

54. Mandelbaum N, Gefen T, Geva-Zatorsky N. Gut bacteria - not for the faint of heart. Cell Host Microbe. 2020;27:1-3. https://doi.org/ 10.1016/j.chom.2019.12.011.

55. Guo L, Ren L, Yang S, Xiao M, Chang D, Yang F, et al. Profiling early humoral response to diagnose novel coronavirus disease (COVID-19). Clin Infect Dis. 2020;71:778-85. https://doi.org/ 10.1093/cid/ciaa310.

56. French MA, Moodley Y. The role of SARS-CoV -2 antibodies in COVID -19: healing in most, harm at times. Respirology. 2020;25:680-2. https://doi.org/10.1111/resp.13852.

57. Lin C, Xiang J, Yan M, Li H, Huang S, Shen C. Comparison of throat swabs and sputum specimens for viral nucleic acid detection in 52 cases of novel coronavirus (SARS-Cov-2)-infected pneumonia (COVID-19). Clin Chem Lab Med CCLM. 2020;58:1089-94. https://doi.org/10.1515/cclm-2020-0187.

58. Li C, Ren L. Recent progress on the diagnosis of 2019 novel coronavirus. Transbound Emerg Dis. 2020. https://doi.org/10. 1111/tbed.13620.

59. Yu L, Wu S, Hao X, Dong X, Mao L, Pelechano V, et al. Rapid detection of COVID-19 coronavirus using a reverse transcriptional loop-mediated isothermal amplification (RT-LAMP) diagnostic platform. Clin Chem. 2020;66:975-7. https://doi.org/10.1093/ clinchem/hvaa102.

60. Yang W, Dang X, Wang Q, Xu M, Zhao Q, Zhou Y, Zhao H, Wang L, Xu Y, Wang J, Han S, Wang M, Pei F, Wang Y (2020) Rapid detection of SARS-CoV-2 using reverse transcription RTLAMP method. infectious diseases (except HIV/AIDS)

61. Huang WE, Lim B, Hsu C, Xiong D, Wu W, Yu Y, et al. RTLAMP for rapid diagnosis of coronavirus SARS-CoV-2. Microb Biotechnol. 2020;13:950-61. https://doi.org/10.1111/1751-7915. 13586.

62. Asefi H, Safaie A. The role of chest CT scan in diagnosis of COVID-19. Adv J Emerg Med. 2020;4. https://doi.org/10. 22114/ajem.v4i2s.451.

63. Li Y, Xia L. Coronavirus disease 2019 (COVID-19): role of Chest CT in diagnosis and management. Am J Roentgenol. 2020;214: 1280-6. https://doi.org/10.2214/AJR.20.22954.

64. Hernandez-Vargas G, Sosa-Hernández JE, Saldarriaga-Hernandez S, Villalba-Rodríguez AM, Parra-Saldivar R, Iqbal H. Electrochemical biosensors: a solution to pollution detection with reference to environmental contaminants. Biosensors. 2018;8:29.

65. Brazaca LC, Ribovski L, Janegitz BC, Zucolotto V. Nanostructured materials and nanoparticles for point of care (POC) medical biosensors. In: Medical Biosensors for Point of Care (POC) Applications: Elsevier; 2017. p. 229-54.

66. Saeed AA, Sánchez JLA, O'Sullivan CK, Abbas MN. DNA biosensors based on gold nanoparticles-modified graphene oxide for the detection of breast cancer biomarkers for early diagnosis. Bioelectrochemistry. 2017;118:91-9.

67. Choi JR, Yong KW, Choi JY, Cowie AC. Emerging point-of-care technologies for food safety analysis. Sensors. 2019;19:817. 
68. Mehrotra P. Biosensors and their applications-a review. J Oral Biol Craniofacial Res. 2016;6:153-9.

69. Wu MY-C, Hsu M-Y, Chen S-J, Hwang D-K, Yen T-H, Cheng CM. Point-of-care detection devices for food safety monitoring: proactive disease prevention. Trends Biotechnol. 2017;35:288300

70. Fraser LA, Kinghorn AB, Dirkzwager RM, Liang S, Cheung YW, Lim B, et al. A portable microfluidic aptamer-tethered enzyme capture (APTEC) biosensor for malaria diagnosis. Biosens Bioelectron. 2018;100:591-6. https://doi.org/10.1016/j.bios. 2017.10.001.

71. Garg N, Boyle D, Randall A, Teng A, Pablo J, Liang X, et al. Rapid immunodiagnostics of multiple viral infections in an acoustic microstreaming device with serum and saliva samples. Lab Chip. 2019;19:1524-33. https://doi.org/10.1039/C8LC01303A.

72. Belushkin A, Yesilkoy F, González-López JJ, Ruiz-Rodríguez JC, Ferrer R, Fàbrega A, et al. Rapid and digital detection of inflammatory biomarkers enabled by a novel portable nanoplasmonic imager. Small. 2020;16:1906108. https://doi.org/10.1002/smll. 201906108

73. Bianchi V, Mattarozzi M, Giannetto M, Boni A, De Munari I, Careri M. A self-calibrating IoT portable electrochemical immunosensor for serum human epididymis protein 4 as a tumor biomarker for ovarian cancer. Sensors. 2020;20:2016. https://doi. org/10.3390/s20072016.

74. Cui X, Hu J, Choi JR, Huang Y, Wang X, Lu TJ, et al. A volumetric meter chip for point-of-care quantitative detection of bovine catalase for food safety control. Anal Chim Acta. 2016;935: 207-12.

75. Zhang J, Chai X, He X-P, Kim H-J, Yoon J, Tian H. Fluorogenic probes for disease-relevant enzymes. Chem Soc Rev. 2019;48: 683-722.

76. Nasseri B, Soleimani N, Rabiee N, Kalbasi A, Karimi M, Hamblin MR. Point-of-care microfluidic devices for pathogen detection. Biosens Bioelectron. 2018;117:112-28.

77. Qiu X, Ge S, Gao P, Li K, Yang S, Zhang S, et al. A smartphonebased point-of-care diagnosis of H1N1 with microfluidic convection PCR. Microsyst Technol. 2017;23:2951-6.

78. Kaarj K, Akarapipad P, Yoon J-Y. Simpler, faster, and sensitive Zika virus assay using smartphone detection of loop-mediated isothermal amplification on paper microfluidic chips. Sci Rep. 2018;8:1-11.

79. Feng S, Choi JR, Lu TJ, Xu F. State-of-art advances in liquid penetration theory and flow control in paper for paper-based diagnosis. Adv Porous Flow. 2015;5:16-29.

80. Gong Y, Hu J, Choi JR, You M, Zheng Y, Xu B, et al. Improved LFIAs for highly sensitive detection of BNP at point-of-care. Int J Nanomedicine. 2017;12:4455

81. Mahato K, Srivastava A, Chandra P. Paper based diagnostics for personalized health care: Emerging technologies and commercial aspects. Biosens Bioelectron. 2017;96:246-59.

82. Afsahi S, Lerner MB, Goldstein JM, Lee J, Tang X, Bagarozzi DA $\mathrm{Jr}$, et al. Novel graphene-based biosensor for early detection of Zika virus infection. Biosens Bioelectron. 2018;100:85-8.

83. Mogha NK, Sahu V, Sharma RK, Masram DT. Reduced graphene oxide nanoribbon immobilized gold nanoparticle based electrochemical DNA biosensor for the detection of Mycobacterium tuberculosis. J Mater Chem B. 2018;6:5181-7.

84. Choi JR, Nilghaz A, Chen L, Chou KC, Lu X. Modification of thread-based microfluidic device with polysiloxanes for the development of a sensitive and selective immunoassay. Sens Actuators B Chem. 2018;260:1043-51.

85. Choi JR, Yong KW, Choi JY, Nilghaz A, Lin Y, Xu J, et al. Black phosphorus and its biomedical applications. Theranostics. 2018;8: 1005 .
86. Kou J, Nguyen EP, Merkoçi A, Guo Z. 2-dimensional materialsbased electrical/optical platforms for smart on-off diagnostics applications. 2D Mater. 2020;7:032001. https://doi.org/10.1088/ 2053-1583/ab896a.

87. Feldmann M, Maini RN, Woody JN, Holgate ST, Winter G, Rowland M, et al. Trials of anti-tumour necrosis factor therapy for COVID-19 are urgently needed. The Lancet. 2020;395:14079. https://doi.org/10.1016/S0140-6736(20)30858-8.

88. Mehta P, McAuley DF, Brown M, Sanchez E, Tattersall RS, Manson JJ. COVID-19: consider cytokine storm syndromes and immunosuppression. The Lancet. 2020;395:1033-4. https://doi. org/10.1016/S0140-6736(20)30628-0.

89. Russell SM, Alba-Patiño A, Barón E, Borges M, Gonzalez-Freire $\mathrm{M}$, de la Rica R. Biosensors for managing the COVID-19 cytokine storm: challenges ahead. ACS Sens. 2020;5:1506-13. https://doi. org/10.1021/acssensors.0c00979.

90. Joh DY, Hucknall AM, Wei Q, Mason KA, Lund ML, Fontes $\mathrm{CM}$, et al. Inkjet-printed point-of-care immunoassay on a nanoscale polymer brush enables subpicomolar detection of analytes in blood. Proc Natl Acad Sci. 2017;114:E7054-62. https://doi.org/ 10.1073/pnas.1703200114.

91. Lin Q, Wen D, Wu J, Liu L, Wu W, Fang X, et al. Microfluidic immunoassays for sensitive and simultaneous detection of $\mathrm{IgG} /$ IgM/antigen of SARS-CoV-2 within $15 \mathrm{~min}$. Anal Chem. 2020;92:9454-8. https://doi.org/10.1021/acs.analchem.0c01635.

92. Sun AC, Yao C, A.g. V, Hall DA. An efficient power harvesting mobile phone-based electrochemical biosensor for point-of-care health monitoring. Sens Actuators B Chem. 2016;235:126-35. https://doi.org/10.1016/j.snb.2016.05.010.

93. Liu Q, Zhou X, Wu H, Zheng B. Blocking-free and self-contained immunoassay platform for one-step point-of-care testing. Biosens Bioelectron. 2020;165:112394. https://doi.org/10.1016/j.bios. 2020.112394.

94. Lee CY-P, Lin RTP, Renia L, Ng LFP. Serological approaches for COVID-19: epidemiologic perspective on surveillance and control. Front Immunol. 2020;11. https://doi.org/10.3389/fimmu. 2020.00879 .

95. Grifoni A, Weiskopf D, Ramirez SI, Mateus J, Dan JM, Moderbacher CR, et al. Targets of $\mathrm{T}$ cell responses to SARSCoV-2 coronavirus in humans with COVID-19 disease and unexposed individuals. Cell. 2020;181:1489-1501.e15. https://doi.org/ 10.1016/j.cell.2020.05.015.

96. Yuan M, Wu NC, Zhu X, Lee C-CD, So RTY, Lv H, et al. A highly conserved cryptic epitope in the receptor binding domains of SARS-CoV-2 and SARS-CoV. Science. 2020;368:630-3. https://doi.org/10.1126/science.abb7269.

97. Cesewski E, Johnson BN. Electrochemical biosensors for pathogen detection. Biosens Bioelectron. 2020;159:112214. https://doi. org/10.1016/j.bios.2020.112214.

98. Cetin AE, Coskun AF, Galarreta BC, Huang M, Herman D, Ozcan $\mathrm{A}$, et al. Handheld high-throughput plasmonic biosensor using computational on-chip imaging. Light Sci Appl. 2014;3:e122. https://doi.org/10.1038/lsa.2014.3.

99. Coskun AF, Cetin AE, Galarreta BC, Alvarez DA, Altug H, Ozcan A. Lensfree optofluidic plasmonic sensor for real-time and label-free monitoring of molecular binding events over a wide field-of-view. Sci Rep. 2014;4:6789. https://doi.org/10.1038/ srep06789.

100. Wang Y, Zhou J, Li J. Construction of plasmonic nano-biosensorbased devices for point-of-care testing. Small Methods. 2017;1: 1700197. https://doi.org/10.1002/smtd.201700197.

101. Peláez EC, Estevez MC, Mongui A, Menéndez M-C, Toro C, Herrera-Sandoval OL, et al. Detection and quantification of HspX antigen in sputum samples using plasmonic biosensing: toward a real point-of-care (POC) for tuberculosis diagnosis. 
ACS Infect Dis. 2020;6:1110-20. https://doi.org/10.1021/ acsinfecdis.9b00502.

102. Brangel P, Sobarzo A, Parolo C, Miller BS, Howes PD, Gelkop S, et al. A serological point-of-care test for the detection of IgG antibodies against Ebola virus in human survivorS. ACS Nano. 2018;12:63-73. https://doi.org/10.1021/acsnano.7b07021.

103. Li Z, Yi Y, Luo X, Xiong N, Liu Y, Li S, et al. Development and clinical application of a rapid IgM-IgG combined antibody test for SARS-CoV-2 infection diagnosis. J Med Virol. 2020;n/a:1-7. https://doi.org/10.1002/jmv.25727.

104. Loo SW, Pui T-S. Cytokine and cancer biomarkers detection: the dawn of electrochemical paper-based biosensor. Sensors. 2020;20:1854. https://doi.org/10.3390/s20071854.

105. Zou Y, Zhang Y, Xu Y, Chen Y, Huang S, Lyu Y, et al. Portable and label-free detection of blood bilirubin with graphene-isolatedAu-nanocrystals paper strip. Anal Chem. 2018;90:13687-94. https://doi.org/10.1021/acs.analchem.8b04058.

106. Lim WY, Thevarajah TM, Goh BT, Khor SM. Paper microfluidic device for early diagnosis and prognosis of acute myocardial infarction via quantitative multiplex cardiac biomarker detection. Biosens Bioelectron. 2019;128:176-85. https://doi.org/10.1016/j. bios.2018.12.049.

107. Tenda K, van Gerven B, Arts R, Hiruta Y, Merkx M, Citterio D. Paper-based antibody detection devices using bioluminescent BRET-switching sensor proteins. Angew Chem Int Ed. 2018;57: 15369-73. https://doi.org/10.1002/anie.201808070.

108. Zhao J, Yuan Q, Wang H, Liu W, Liao X, Su Y, et al. Antibody responses to SARS-CoV-2 in patients of novel coronavirus disease 2019. Clin Infect Dis. 2020. https://doi.org/10.1093/cid/ ciaa344.

109. Soum V, Park S, Brilian AI, Kwon O-S, Shin K. Programmable paper-based microfluidic devices for biomarker detections. Micromachines. 2019;10:516. https://doi.org/10.3390/ mi10080516.

110. Amin N, Torralba AS, Álvarez-Diduk R, Afkhami A, Merkoçi A. Lab in a tube: point-of-care detection of Escherichia coli. Anal Chem. 2020;92:4209-16. https://doi.org/10.1021/acs.analchem. $9 \mathrm{~b} 04369$.

111. Tao D, Gu Y, Song S, Nguyen EP, Cheng J, Yuan Q, et al. Ultrasensitive detection of alpha-synuclein oligomer using a PolyD-glucosamine/gold nanoparticle/carbon-based nanomaterials modified electrochemical immunosensor in human plasma. Microchem J. 2020;158:105195. https://doi.org/10.1016/ j.microc.2020.105195.

112. Zhao Y, Liu H, Shi L, Zheng W, Jing X. Electroactive Cu2O nanoparticles and $\mathrm{Ag}$ nanoparticles driven ratiometric electrochemical aptasensor for prostate specific antigen detection. Sens Actuators B Chem. 2020;315:128155. https://doi.org/10.1016/j. snb.2020.128155.

113. Chen Y, Xianyu Y, Wu J, Dong M, Zheng W, Sun J, et al. Doubleenzymes-mediated bioluminescent sensor for quantitative and ultrasensitive point-of-care testing. Anal Chem. 2017;89:5422-7. https://doi.org/10.1021/acs.analchem.7b00239.

114. Gao A, Yang X, Tong J, Zhou L, Wang Y, Zhao J, et al. Multiplexed detection of lung cancer biomarkers in patients serum with CMOS-compatible silicon nanowire arrays. Biosens Bioelectron. 2017;91:482-8. https://doi.org/10.1016/j.bios.2016. 12.072 .

115. Mayorga-Martinez CC, Mohamad Latiff N, Eng AYS, Sofer Z, Pumera M. Black phosphorus nanoparticle labels for immunoassays via hydrogen evolution reaction mediation. Anal Chem. 2016;88:10074-9.

116. Ambhorkar P, Wang Z, Ko H, Lee S, Koo K, Kim K, et al. Nanowire-based biosensors: from growth to applications. Micromachines. 2018;9:679. https://doi.org/10.3390/mi9120679.
117. Doucey M-A, Carrara S. Nanowire sensors in cancer. Trends Biotechnol. 2019;37:86-99. https://doi.org/10.1016/j.tibtech. 2018.07.014.

118. Novoselov KS, Geim AK, Morozov SV, Jiang D, Zhang Y, Dubonos SV, et al. Electric field effect in atomically thin carbon films. Science. 2004;306:666-9. https://doi.org/10.1126/science. 1102896.

119. Bolotsky A, Butler D, Dong C, Gerace K, Glavin NR, Muratore C, et al. Two-dimensional materials in biosensing and healthcare: from in vitro diagnostics to optogenetics and beyond. ACS Nano. 2019;13:9781-810. https://doi.org/10.1021/acsnano. $9 \mathrm{~b} 03632$.

120. Hatamie A, Angizi S, Kumar S, Pandey CM, Simchi A, Willander $\mathrm{M}$, et al. Review - textile based chemical and physical sensors for healthcare monitoring. J Electrochem Soc. 2020;167:037546. https://doi.org/10.1149/1945-7111/ab6827.

121. He W, Wang C, Wang H, Jian M, Lu W, Liang X, et al. Integrated textile sensor patch for real-time and multiplex sweat analysis. Sci Adv. 2019;5:eaax0649. https://doi.org/10.1126/sciadv.aax0649.

122. Noviana E, Jain S, Hofstetter J, Geiss BJ, Dandy DS, Henry CS. based nuclease protection assay with on-chip sample pretreatment for point-of-need nucleic acid detection. Anal Bioanal Chem. 2020:1-11.

123. Ozer T, Geiss BJ, Henry CS. Chemical and biological sensors for viral detection. J Electrochem Soc. 2019;167:037523.

124. Zhang S, Su X, Wang J, Chen M, Li C, Li T, et al. Nucleic acid testing for coronavirus disease 2019: demand, research progression, and perspective. Crit Rev Anal Chem. 2020:1-12.

125. Jalandra R, Yadav AK, Verma D, Dalal N, Sharma M, Singh R, et al. Strategies and perspectives to develop SARS-CoV-2 detection methods and diagnostics. Biomed Pharmacother. 2020: 110446.

126. van Kasteren PB, van der Veer B, van den Brink S, Wijsman L, de Jonge J, van den Brandt A, et al. Comparison of commercial RTPCR diagnostic kits for COVID-19. J Clin Virol. 2020;104412.

127. Tang RH, Liu LN, Zhang SF, He XC, Li XJ, Xu F, et al. A review on advances in methods for modification of paper supports for use in point-of-care testing. Microchim Acta. 2019;186:521.

128. Khan M, Hasan M, Hossain S, Ahommed M, Daizy M. Ultrasensitive detection of pathogenic viruses with electrochemical biosensor: state of the art. Biosens Bioelectron. 2020;112431.

129. Khan NI, Song E. Lab-on-a-chip systems for aptamer-based biosensing. Micromachines. 2020;11:220.

130. Sadighbayan D, Sadighbayan K, Khosroushahi AY, Hasanzadeh M. Recent advances on the DNA-based electrochemical biosensing of cancer biomarkers: analytical approach. TrAC Trends Anal Chem. 2019;119:115609.

131. Sri S, Dhand C, Rathee J, Ramakrishna S, Solanki PR. Microfluidic based biosensors as point of care devices for infectious diseases management. Sens Lett. 2019;17:4-16.

132. Zhu H, Zhang H, Ni S, Korabečná M, Yobas L, Neuzil P. The vision of point-of-care PCR tests for the COVID-19 pandemic and beyond. TrAC Trends Anal Chem. 2020;115984.

133. Gonzalez-Gonzalez E, Lara-Mayorga IM, Rodriguez-Sanchez IP, Yee-de Leon F, Garcia-Rubio A, Garciamendez-Mijares CE, et al. Scaling diagnostics in times of COVID-19: colorimetric loopmediated isothermal amplification (LAMP) assisted by a 3Dprinted incubator for cost-effective and scalable detection of SARS-CoV-2. medRxiv. 2020.

134. Sun F, Ganguli A, Nguyen J, Brisbin R, Shanmugam K, Hirschberg DL, et al. Smartphone-based multiplex 30-minute nucleic acid test of live virus from nasal swab extract. Lab Chip. 2020;20:1621-7.

135. Ganguli A, Mostafa A, Berger J, Aydin M, Sun F, Valera E, et al. Rapid isothermal amplification and portable detection system for SARS-CoV-2. bioRxiv. 2020. 
136. Dou M, Sanjay ST, Dominguez DC, Liu P, Xu F, Li X. Multiplexed instrument-free meningitis diagnosis on a polymer/ paper hybrid microfluidic biochip. Biosens Bioelectron. 2017;87: 865-73.

137. Dou M, Sanchez J, Tavakoli H, Gonzalez JE, Sun J, Bard JD, et al. A low-cost microfluidic platform for rapid and instrument-free detection of whooping cough. Anal Chim Acta. 2019;1065:71-8.

138. Malpartida-Cardenas K, Miscourides N, Rodriguez-Manzano J, Yu L-S, Moser N, Baum J, et al. Quantitative and rapid Plasmodium falciparum malaria diagnosis and artemisininresistance detection using a CMOS Lab-on-Chip platform. Biosens Bioelectron. 2019;145:111678.

139. Rodriguez-Manzano J, Malpartida-Cardenas K, Moser N, Pennisi I, Cavuto M, Miglietta L, et al. A handheld point-of-care system for rapid detection of SARS-CoV-2 in under 20 minutes. medRxiv. 2020.

140. Broughton JP, Deng X, Yu G, Fasching CL, Servellita V, Singh J, et al. CRISPR-Cas12-based detection of SARS-CoV-2. Nat Biotechnol. 2020:1-5.

141. Tripathy S, Singh SG. Label-free electrochemical detection of DNA hybridization: a method for COVID-19 diagnosis. Trans Indian Natl Acad Eng. 2020;1.

142. Tripathy S, Joseph J, Vanjari SRK, Rao AN, Singh SG. Flexible ITO electrode with gold nanostructures for femtomolar DNA hybridization detection. IEEE Sens Lett. 2018;2:1-4.

143. Tripathy S, Gangwar R, Supraja P, Rao AN, Vanjari SRK, Singh SG. Graphene doped $\mathrm{Mn} 2 \mathrm{O} 3$ nanofibers as a facile electroanalytical DNA point mutation detection platform for early diagnosis of breast/ovarian cancer. Electroanalysis. 2018;30:2110-20.

144. Anker JN, Hall WP, Lyandres O, Shah NC, Zhao J, Van Duyne RP. Biosensing with plasmonic nanosensors. In: Nanoscience and Technology: A Collection of Reviews from Nature Journals: World Scientific; 2010. p. 308-19.

145. Qiu G, Gai Z, Tao Y, Schmitt J, Kullak-Ublick GA, Wang J. Dualfunctional plasmonic photothermal biosensors for highly accurate severe acute respiratory syndrome coronavirus 2 detection. ACS Nano. 2020;14:5268-77.

146. Ozer T, McMahon C, Henry CS. Advances in paper-based analytical devices. Annu Rev Anal Chem. 2020;13.

147. Zhang D, Broyles D, Hunt EA, Dikici E, Daunert S, Deo SK. A paper-based platform for detection of viral RNA. Analyst. 2017;142:815-23.

148. Zhu X, Wang X, Han L, Chen T, Wang L, Li H, et al. Multiplex reverse transcription loop-mediated isothermal amplification combined with nanoparticle-based lateral flow biosensor for the diagnosis of COVID-19. Biosens Bioelectron. 2020:112437.

149. Choi JR, Hu J, Tang R, Gong Y, Feng S, Ren H, et al. An integrated paper-based sample-to-answer biosensor for nucleic acid testing at the point of care. Lab Chip. 2016;16:611-21.

150. Abduljalil JM. Laboratory diagnosis of SARS-CoV-2: available approaches and limitations. New Microbes New Infect. 2020;100713.

151. Li Y, Li S, Wang J, Liu G. CRISPR/Cas systems towards nextgeneration biosensing. Trends Biotechnol. 2019;37:730-43.

152. Wang X, Zhong M, Liu Y, Ma P, Dang L, Meng Q, et al. Rapid and sensitive detection of COVID-19 using CRISPR/Cas12abased detection with naked eye readout, CRISPR/Cas12a-NER. Sci Bull. 2020.

153. Lucia C, Federico P-B, Alejandra GC. An ultrasensitive, rapid, and portable coronavirus SARS-CoV-2 sequence detection method based on CRISPR-Cas12. bioRxiv. 2020.

154. Yoshimi K, Takeshita K, Yamayoshi S, Shibumura S, Yamauchi Y, Yamamoto M, et al. Rapid and accurate detection of novel coronavirus SARS-CoV-2 using CRISPR-Cas3. medRxiv. 2020.
155. Joung J, Ladha A, Saito M, Segel M, Bruneau R, Huang MW, et al. Point-of-care testing for COVID-19 using SHERLOCK diagnostics. medRxiv. 2020.

156. Rodriguez NM, Wong WS, Liu L, Dewar R, Klapperich CM. A fully integrated paperfluidic molecular diagnostic chip for the extraction, amplification, and detection of nucleic acids from clinical samples. Lab Chip. 2016;16:753-63.

157. Rodriguez NM, Linnes JC, Fan A, Ellenson CK, Pollock NR, Klapperich CM. based RNA extraction, in situ isothermal amplification, and lateral flow detection for low-cost, rapid diagnosis of influenza A (H1N1) from clinical specimens. Anal Chem. 2015;87:7872-9.

158. Tian B, Gao F, Fock J, Dufva M, Hansen MF. Homogeneous circle-to-circle amplification for real-time optomagnetic detection of SARS-CoV-2 RdRp coding sequence. Biosens Bioelectron. 2020;112356.

159. Gong Y, Zheng Y, Jin B, You M, Wang J, Li X, et al. A portable and universal upconversion nanoparticle-based lateral flow assay platform for point-of-care testing. Talanta. 2019;201:126-33.

160. Du T-E, Wang Y, Zhang Y, Zhang T, Mao X. A novel adenosinebased molecular beacon probe for room temperature nucleic acid rapid detection in cotton thread device. Anal Chim Acta. 2015;861:69-73.

161. Bao M, Jensen E, Chang Y, Korensky G, Du K. Magnetic beadquantum dot (MB-Qdot) CRISPR assay for instrument-free viral DNA detection. bioRxiv. 2020.

162. Papadakis G, Pantazis AK, Fikas N, Chatziioannidou S, Michaelidou K, Pogka V, et al. Real-time colorimetric LAMP methodology for quantitative nucleic acids detection at the point-of-care. bioRxiv. 2020.

163. Weng X, Kang Y, Guo Q, Peng B, Jiang H. Recent advances in thread-based microfluidics for diagnostic applications. Biosens Bioelectron. 2019;132:171-85.

164. Sun K, Xing W, Yu X, Fu W, Wang Y, Zou M, et al. Recombinase polymerase amplification combined with a lateral flow dipstick for rapid and visual detection of Schistosoma japonicum. Parasit Vectors [Internet]. 2016 Aug 31 [cited 2020 Sep 20];9(1):476. Available from: https://doi.org/10.1186/s13071-016-1745-5.

165. Yan WX, Hunnewell P, Alfonse LE, Carte JM, Keston-Smith E, Sothiselvam S, et al. Functionally diverse type V CRISPR-Cas systems. Science. 2019;363(6422):88-91.

166. Ma Q, Liu H, Ye F, Xiang G, Shan W, Xing W. Rapid and visual detection of Mycobacterium tuberculosis complex using recombinase polymerase amplification combined with lateral flow strips. Mol Cell Probes [Internet]. 2017 Dec 1 [cited 2020 Sep 20];36:43-49. Available from: http://www.sciencedirect.com/ science/article/pii/S0890850817300804.

167. Li Z, Yi Y, Luo X, Xiong N, Liu Y, Li S, et al. Development and clinical application of a rapid IgM-IgG combined antibody test for SARS-CoV-2 infection diagnosis. J Med Virol. 2020.

168. Haymond A, Mueller C, Steinberg H, Hodge KA, Lehman CW, Lin S-C, et al. Clinical utility of a highly sensitive lateral flow immunoassay as determined by titer analysis for the detection of anti-SARS-CoV-2 antibodies at the point-of-care. medRxiv. 2020.

169. Black MA, Shen G, Feng X, Beltran WG, Feng Y, Vasudevaraja $\mathrm{V}$, et al. Analytical performance of lateral flow immunoassay for SARS-CoV-2 exposure screening on venous and capillary blood samples. medRxiv. 2020.

170. Charlton CL, Kanji JN, Johal K, Bailey A, Plitt SS, MacDonald C, et al. Evaluation of six commercial mid to high volume antibody and six point of care lateral flow assays for detection of SARSCoV-2 antibodies. J Clin Microbiol. 2020.

171. Mueller L, Ostermann PN, Walker A, Wienemann T, Mertens A, Adams O, et al. Sensitivity of commercial Anti-SARS-CoV-2 serological assays in a high-prevalence setting. medRxiv. 2020. 
172. Weidner L, Gänsdorfer S, Unterweger S, Weseslindtner L, Drexler C, Farcet M, et al. Quantification of SARS-CoV-2 antibodies with eight commercially available immunoassays. J Clin Virol. 2020;104540.

173. Wölfel R, Corman VM, Guggemos W, Seilmaier M, Zange S, Müller MA, et al. Virological assessment of hospitalized patients with COVID-2019. Nature. 2020;581(7809):465-9.

174. Choi JR, Nilghaz A, Chen L, Chou KC, Lu X. Modification of thread-based microfluidic device with polysiloxanes for the development of a sensitive and selective immunoassay. Sens Actuators B Chem. 2018 May 1;260:1043-51.

175. Favara DM, Cooke A, Doffinger R, Houghton S, Budriunaite I, Bossingham S, et al. First results from the UK COVID-19 Serology in Oncology Staff study (CSOS). medRxiv. 2020.

176. Bond K, Nicholson S, Hoang T, Catton M, Howden B, Williamson D. Post-market validation of three serological assays for COVID-19. Doherty Inst. 2020.

177. Walker FM, Hsieh K. Advances in directly amplifying nucleic acids from complex samples. Biosensors. 2019;9:117.

178. Wang T-M, Yang J-T. Visual DNA diagnosis of tomato yellow leaf curl virus with integrated recombinase polymerase amplification and a gold-nanoparticle probe. Sci Rep. 2019;9:1-8.

179. Linares CA, Ryan F, Moses SE. Early data on the performance of a combined SARS-CoV-2 spike-nucleocapsid antibody lateral flow device compared to a nucleocapsid-only device. bioRxiv. 2020.

180. Pallett SJ, Rayment M, Patel A, Fitzgerald-Smith SA, Denny SJ, Charani E, et al. Point-of-care serological assays for delayed SARS-CoV-2 case identification among health-care workers in the UK: a prospective multicentre cohort study. Lancet Respir Med. 2020.

181. Hoffman T, Nissen K, Krambrich J, Rönnberg B, Akaberi D, Esmaeilzadeh M, et al. Evaluation of a COVID-19 IgM and IgG rapid test; an efficient tool for assessment of past exposure to SARS-CoV-2. Infect Ecol Epidemiol. 2020;10:1754538. https:// doi.org/10.1080/20008686.2020.1754538.

182. Choi JR, Liu Z, Hu J, Tang R, Gong Y, Feng S, et al. Polydimethylsiloxane-paper hybrid lateral flow assay for highly sensitive point-of-care nucleic acid testing. Anal Chem. 2016;88: 6254-64. https://doi.org/10.1021/acs.analchem.6b00195.

183. Gao X, Xu L-P, Wu T, Wen Y, Ma X, Zhang X. An enzymeamplified lateral flow strip biosensor for visual detection of
MicroRNA-224. Talanta. 2016;146:648-54. https://doi.org/10. 1016/j.talanta.2015.06.060.

184. Tang R, Yang H, Choi JR, Gong Y, Hu J, Feng S, et al. Improved sensitivity of lateral flow assay using paper-based sample concentration technique. Talanta. 2016;152:269-76. https://doi.org/10. 1016/j.talanta.2016.02.017.

185. Li J, Macdonald J. Multiplexed lateral flow biosensors: technological advances for radically improving point-of-care diagnoses. Biosens Bioelectron. 2016;83:177-92. https://doi.org/10.1016/j. bios.2016.04.021.

186. Choi JR, Yong KW, Tang R, Gong Y, Wen T, Li F, et al. Advances and challenges of fully integrated paper-based pointof-care nucleic acid testing. TrAC Trends Anal Chem. 2017;93: 37-50. https://doi.org/10.1016/j.trac.2017.05.007.

187. Blicharz TM, Gong P, Bunner BM, Chu LL, Leonard KM, Wakefield JA, et al. Microneedle-based device for the one-step painless collection of capillary blood samples. Nat Biomed Eng. 2018;2:151-7.

188. Aro K, Wei F, Wong DT, Tu M. Saliva liquid biopsy for point-ofcare applications. Front Public Health. 2017;5:77.

189. Deng J, Jiang X. Advances in reagents storage and release in selfcontained point-of-care devices. Adv Mater Technol. 2019;4: 1800625. https://doi.org/10.1002/admt.201800625.

190. Montes-Cebrián Y, del Torno-de Román L, Álvarez-Carulla A, Colomer-Farrarons J, Minteer SD, Sabaté N, et al. 'Plug-and-power' point-of-care diagnostics: a novel approach for self-powered electronic reader-based portable analytical devices. Biosens Bioelectron. 2018;118:88-96. https://doi.org/10.1016/j.bios. 2018.07.034

191. Xu D, Huang X, Guo J, Ma X. Automatic smartphone-based microfluidic biosensor system at the point of care. Biosens Bioelectron. 2018;110:78-88. https://doi.org/10.1016/j.bios. 2018.03.018.

192. Yang T, Gentile M, Shen C-F, Cheng C-M. Combining point-ofcare diagnostics and internet of medical things (IoMT) to combat the COVID-19 pandemic. Diagnostics. 2020;10:224. https://doi. org/10.3390/diagnostics10040224.

Publisher's note Springer Nature remains neutral with regard to jurisdictional claims in published maps and institutional affiliations. 